\title{
Turbulence Model and Numerical Scheme Assessment for Buffet Computations
}

Eric GONCALVES

LEGI

BP 53, 38041 Grenoble cedex 9, France

Eric.Goncalves@hmg.inpg.fr

\section{Robert HOUDEVILLE}

ONERA-Toulouse, Department of Aerodynamics and Energetics, BP 4025, Toulouse, 31055 cedex, France

Robert.Houdeville@onecert.fr

\begin{abstract}
The prediction of shock-induced oscillations over transonic rigid airfoils is important for a better understanding of the buffeting phenomenon. The unsteady resolution of the NavierStokes equations is performed with various transport-equation turbulence models in which corrections are added for non-equilibrium flows. The lack of numerical efficiency due to the CFL stability condition is circumvented by the use of a wall law approach and a dual time stepping method. Moreover, various numerical schemes are used to try and be independent of the numerical discretization.

Comparisons are made with the experimental results obtained for the supercritical RA16SC1 airfoil. They show the interest in using the SST correction or realizability conditions to get correct predictions of the frequency, amplitude and pressure fluctuations over the airfoil.
\end{abstract}

\section{Keywords}

Buffet, Unsteady Flows, Turbulence Modelling, Numerical Scheme 


\section{Nomenclature}

$x, y, z \quad$ local wall frame (boundary layer)

$U_{\tau} \quad$ friction velocity

$C_{f} \quad$ shear stress coefficient

$C_{p} \quad$ pressure coefficient

$F_{c}, F_{d} \quad$ convective and diffusive flux densities

$P_{k} \quad$ turbulent kinetic energy production

$M_{\infty} \quad$ infinite Mach number

$R e_{c} \quad$ Reynolds number based on the mean chord

$T_{i} \quad$ stagnation temperature

$u, v, w \quad$ velocity components in the local wall frame

$q \quad$ total heat flux, $q^{v}+q^{t}$

$k \quad$ turbulent kinetic energy

$P \quad$ static pressure

$P_{r}, P_{r t} \quad$ Prandtl numbers

$T$ mean static temperature

$\alpha \quad$ angle of attack

$\varepsilon \quad$ dissipation rate

$\kappa \quad$ von Karman constant

$\omega \quad$ specific dissipation

$\mu, \mu_{t} \quad$ molecular and eddy viscosity

$\rho \quad$ density

$\overline{\bar{\tau}} \quad$ total stress tensor, $\overline{\overline{\tau^{v}}}+\overline{\overline{\tau^{t}}}$

$w \quad$ wall value

$+\quad$ wall scale

1 adjacent cell with respect to the wall

$v \quad$ viscous

$t \quad$ turbulent 


\section{INTRODUCTION}

The numerical simulation of unsteady turbulent flows around airfoils is motivated by the need to better understand complex flow phenomena appearing in aeronautic applications such as flows over aircraft wings. The present work focuses on the transonic buffet. This aerodynamic phenomenon results in a self-sustained periodic motion of the shock wave over the surface of the airfoil, due to the development of instabilities caused by the boundary layer separation and the shock wave interaction. The shock-induced oscillations (SIO) over rigid airfoils in transonic regime have been classified by Tijdeman for forced instabilities using a moving trailing edge flap [1]. A detailed description of the physical features of SIO is given by Lee [2]. This problem is of primary importance for aeronautic applications as it can lead to the buffeting phenomenon through the mechanical response of the wing structure. The large amplitude periodic variation of lift associated with buffet limits the cruising speed of commercial aircraft and severely degrades the maneuverability of combat aircraft. Accurate predictions of such flow phenomena is of significant technological interest and their simulation remains a challenging problem due to the complex physics involved.

Today, despite the fast improvement of computer performances, the unsteady resolution of the Navier-Stokes equations remains a difficult problem. Three-dimensional timedependent computations obtained with large eddy simulations (LES) and especially with direct simulations (DNS) are not yet practical for this kind of applications because of the high demands in computer resources. In this study, the Reynolds decomposition was used with an averaged statistical processing resulting in the RANS equations for the mean flow quantities. This approach leads to a low frequency separation between modeled and computed scales. It is well known that these equations can be legitimely used for flows in which the time scale of the mean flow unsteadiness is much larger than the characteristic time scale of the turbulence. This is the case with the transonic buffet in which the shockinduced oscillation frequency is around $100 \mathrm{~Hz}$.

The Reynolds decomposition introduces additional unknown quantities like the Reynolds stress tensor and requires a turbulence model to close the equation system. Various turbulence closures can be found in the literature of unsteady numerical simulation flows associated with buffet, oscillating airfoils or dynamic stall. Models are more or less sophis- 
ticated, from the Baldwin-Lomax algebraic model [3, 4], to one or two transport-equation models [5, 6, 7, 8, 9], to EARSM [8], RSM [9] and non-linear models [9]. Regarding the shock location for steady flows, algebraic models cannot give predictions with an acceptable level of accuracy. The standard eddy-viscosity models based on the linear Boussinesq relation are known to be afflicted by numerous weaknesses, including seriously excessive generation of turbulence at impingement zones, an inability to capture the boundary layer separation and a violation of realizability at large rates of strain. Moreover, theses models are formulated following the spectral energy of Kolmogorov with an equilibrium assumption of turbulence and they are calibrated for steady flows. However, for unsteady flows, the presence of coherent structures can break this equilibrium and lead to a different energy distribution. An observed consequence is the over-production of eddy-viscosity, which limits the unsteadiness development and modifies the flow topology. The present study investigated some corrections for standard linear models such as the shear stree transport (SST) Menter correction and the use of realizability constraints. A first study was conducted, consisting of numerical simulation of transonic buffet over airfoils [10] with the SST correction. It were shown the great influence of this limiter for two-equation models and good results were obtained. Other ways of limiting the eddy-viscosity or the production of turbulence kinetic energy can be used, such as a decrease the value of the $C_{\mu}$ coefficient [12] or the introduction of the vorticity in the production term [13] but they were not tested.

Another important aspect concerns the numerical methods and the computer cost. Indeed, unsteady RANS computations with turbulence models remain expensive. Explicit methods solved the equations using a global time step computed as the minimum of the local time step associated with each grid cell. The CFL stability criterion drastically reduces the method efficiency for fine meshes for which the dimensionless mesh size at the wall must be of unity order, in wall units. To overcome this difficulty, a wall law approach is used to relax the mesh refinement near the wall [11]. Moreover, computations are performed with an efficient implicit method allowing some large time steps and with the dual time-stepping approach allowing the use of acceleleration techniques such as multigrid algorithm and local time step. Finally, the paper presents a numerical scheme comparison to study the influence of the scheme on these unsteady computations and to try and be independent of the spatial discretization. 


\section{NUMERICS}

The numerical simulations were carried out using an implicit CFD code solving the uncoupled RANS/turbulent systems for multi-domain structured meshes. This solver is based on a cell-centered finite-volume discretization.

\section{$2.1 \quad$ Governing equations}

The compressible RANS equations coupled with a two-equation turbulence model in integral form are written for a cell of volume $\Omega$ limited by a surface $\Sigma$ and with an outer normal $n$. These equations can be expressed as :

$$
\begin{gathered}
\frac{d}{d t} \int_{\Omega} w d \Omega+\oint_{\Sigma} F_{c} \cdot n d \Sigma-\oint_{\Sigma} F_{d} \cdot n d \Sigma=\int_{\Omega} S d \Omega \\
w=\left(\begin{array}{c}
\rho \\
\rho V \\
\rho E \\
\rho k \\
\rho \Psi
\end{array}\right) ; \quad F_{c}=\left(\begin{array}{c}
\rho V \\
\rho V \otimes V+p \overline{\bar{I}} \\
(\rho E+p) V \\
\rho k V \\
\rho \Psi V
\end{array}\right) \quad ; \quad F_{d}=\left(\begin{array}{c}
0 \\
\overline{\overline{\tau^{v}}}+\overline{\overline{\tau^{t}}} \\
\left(\overline{\overline{\tau^{v}}}+\overline{\overline{\tau^{t}}}\right) \cdot V-q^{v}-q^{t} \\
\left(\mu+\mu_{t} / \sigma_{k}\right) \operatorname{grad} k \\
\left(\mu+\mu_{t} / \sigma_{\Psi}\right) \operatorname{grad} \Psi
\end{array}\right)
\end{gathered}
$$

where $w$ denotes the conservative variables, $F_{c}$ and $F_{d}$ the convective and diffusive flux densities and $\mathrm{S}$ the source terms which concern only the transport equations. $\Psi$ is the length scale determining variable.

The exact expression of the eddy viscosity $\mu_{t}$ and the source terms depends on the turbulence model, as well as the constants $\sigma_{k}$ and $\sigma_{\Psi}$.

The total stress tensor $\overline{\bar{\tau}}$ is evaluated following the Stokes hypothesis and the Boussinesq assumption. The total heat flux vector $q$ is obtained from the Fourier law with the constant Prandtl number hypothesis.

$$
\begin{aligned}
\overline{\bar{\tau}} & =\overline{\overline{\tau^{v}}}+\overline{\overline{\tau^{t}}}=\left(\mu+\mu_{t}\right)\left[\frac{1}{2}\left(\operatorname{grad} V+(\operatorname{grad} V)^{t}\right)-\frac{2}{3}(\operatorname{div} V) \overline{\bar{I}}\right]+\frac{2}{3} k \overline{\bar{I}} \\
q & =q^{v}+q^{t}=-\left(\frac{\mu}{P_{r}}+\frac{\mu_{t}}{P_{r t}}\right) C_{p} \operatorname{grad} T
\end{aligned}
$$




\subsection{Numerical methods}

For the mean flow, the space-centered Jameson scheme [14] was used. It was stabilized by a scalar artificial dissipation consisting of a blend of $2^{\text {nd }}$ and $4^{\text {th }}$ differences. For the turbulence transport equations, the upwind Roe scheme [15] was used to obtain a more robust method. The second-order accuracy was obtained by introducing a flux-limited dissipation [16]. The Harten's entropy correction was used.

Time integration was performed through a matrix-free implicit method [17, 18]. The implicit method consists in solving a system of equations arising from the linearization of a fully implicit scheme, at each time step. The main feature of this method is that the storage of the Jacobian matrix is completely eliminated, which leads to a low-storage algorithm. The viscous flux Jacobian matrices are replaced by their spectral radii. The convective flux are written with the Roe scheme instead of the Jameson scheme because of the dissipation term, the use of an inconsistent linearization having no consequence for steady computations. The Jacobian matrices which appear from the linearization of the centered fluxes are approximated with the numerical fluxes and the numerical dissipation matrices are replaced by their spectral radii.

Concerning the turbulence transport equations, the diffusive flux Jacobian matrix are also replaced by their spectral radii. The source term needs a special treatment [19]. Only the negative part of the source term Jacobian matrix is considered and replaced by its spectral radius.

The implicit time-integration procedure leads to a system which can be solved directly or iteratively. The direct inversion can be memory intensive and computationally expensive. Therefore, an implicit relaxation procedure is preferred and the point Jacobi relaxation algorithm was chosen.

For steady state computations, convergence acceleration was obtained using a local time step and the full approximation storage (FAS) multigrid method proposed by Jameson [20, 21]. Forcing functions are defined on the coarser grids and added to the residuals used for the stepping scheme. The corrections computed on each coarse grid are transferred back to the finer one by trilinear interpolations. The turbulent equations are only solved on the fine grid and the computed eddy viscosity $\mu_{t}$ is transferred to the coarse grids. The 
multigrid algorithm is applied through a $\mathrm{V}$ type cycle.

For unsteady computations, the dual time stepping method, proposed by Jameson [21], was used to tackle the lack of numerical efficiency of the global time stepping approach. The derivative with respect to the physical time is discretized by a second-order formula. Making the scheme implicit with respect to the dual time provides fast convergence to the time-accurate solution. Between each time step, the solution is advanced in a dual time and acceleration strategies developed for steady problems can be used to speed up the convergence in fictitious time. The initialization of the derivative with respect to the physical time was performed with a first-order formula.

\subsection{Far field conditions}

At the outer edge of the computational domain, a non-reflecting condition is used with a vorticity correction in order to simulate a uniform infinite flow. It is deduced from the flow field induced by a single vortex, the strength of which is given by the airfoil lift [22].

\subsection{Turbulence Models}

Various popular two-equation turbulence models were used in the present study : the Smith $k-l$ model $[23,24]$, the Wilcox $k-\omega$ model [25], the Menter SST $k-\omega$ model $[26,27]$, the high Reynolds version of the Jones-Launder $k-\varepsilon$ model [28], the Kok $k-\omega$ model [29] and also the one-equation Spalart-Allmaras model [30, 31].

As the discretization scheme does not insure the positivity of the turbulent conservative variables, limiters were used to avoid negative $k$ or $\Psi$ values. These limiters were set equal to the corresponding imposed boundary values in the far field.

\section{SST correction}

The Menter correction is based on the empirical Bradshaw's assumption which binds the shear stress to the turbulent kinetic energy for two-dimensional boundary layer. This correction was extended for the $k-\varepsilon$ model and the $k-l$ model. 


\section{non-equilibrium correction}

The nonequilibrium correction of Smith [32], developed for the $k-l$ model, consists in modifying the computation of the eddy viscosity by introducing a function $\sigma$ :

$$
\mu_{t}=\sigma \mu_{t_{e q}} \quad ; \quad \sigma=\frac{\alpha-0.25 \alpha^{1 / 2}+0.875}{\alpha^{3 / 2}+0.625} \quad ; \quad \alpha=\frac{\min \left(P_{k_{e q}}, 0\right)}{\varepsilon}
$$

where the subscript $e q$ denotes the equilibrium value. The non-equilibrium function was chosen to limit the eddy-viscosity when production is greater than dissipation and to increase the viscosity above the equilibrium model value in the contrary case.

\section{Durbin correction - link with realizability}

Based on the realizability principle (the variance of the fluctuating velocity components should be positive and the cross-correlations bounded by the Schwartz inequality), a minimal correction was derived for two-equation turbulence models and was shown to cure the stagnation-point anomaly [33]. The condition to ensure realizability in a three-dimensional flow is :

$$
C_{\mu} \leq \frac{1}{s \sqrt{3}} \quad ; \quad s=\frac{k}{\varepsilon} S \quad ; \quad S^{2}=2 S_{i j} S_{i j}-\frac{2}{3} S_{k k}^{2}
$$

A weakly non-linear model was thus obtained [35] with a $C_{\mu}$ coefficient function of the dimensionless mean strain rate :

$$
C_{\mu}=\min \left(C_{\mu}^{o}, \frac{c}{s \sqrt{3}}\right) \quad \text { with } \quad c \leq 1
$$

where $C_{\mu}^{o}$ is set to the constant value 0.09. Durbin fixed the value of the constant $c$ to 0.5 for good results in impinging jets [34]. Then, the following relation was obtained for the $k-\varepsilon$ model :

$$
\mu_{t}=\rho C_{\mu} \frac{k^{2}}{\varepsilon} \quad ; \quad C_{\mu}=\min \left(C_{\mu}^{o}, \frac{0.3}{s}\right)
$$

And for the $k-\omega$ model :

$$
\mu_{t}=\rho C_{\mu} \frac{k}{\omega} \quad ; \quad C_{\mu}=\min \left(1, \frac{0.3}{C_{\mu}^{o} s}\right)
$$

It should be noted that this correction is similar to the SST formula by replacing $\Omega$ with $S$. Yet, the Durbin correction is established with mathematical concepts and is available for three-dimensional flows whereas the SST correction is based on an empirical twodimensional hypothesis. This model has been successfully tested on shock wave/boundary layer interactions with the Wilcox $k-\omega$ model [35]. 


\section{Recalibration of the constants for the Kok model}

The Kok model has been built in order to resolve the dependence on freestream values of $\omega$. The turbulence transport equations of the model are given by :

$$
\begin{gathered}
\frac{\partial \rho k}{\partial t}+\operatorname{div}\left[\rho k \mathbf{V}-\left(\mu+\sigma_{k} \mu_{t}\right) \operatorname{grad} k\right]=P_{k}-\beta^{*} \rho k \omega \\
\frac{\partial \rho \omega}{\partial t}+\operatorname{div}\left[\rho \omega \mathbf{V}-\left(\mu+\sigma_{\omega} \mu_{t}\right) \operatorname{grad} \omega\right]=P_{\omega}-\beta \rho \omega^{2} \\
+\sigma_{d} \frac{\rho}{\omega} \operatorname{grad} k \cdot \operatorname{grad} \omega
\end{gathered}
$$

Kok obtained additional constraints for the constants :

$$
\begin{array}{r}
\sigma_{\omega}-\sigma_{k}+\sigma_{d}>0 \\
\sigma_{k}-\sigma_{d}>0
\end{array}
$$

The choice of Kok was :

$$
\sigma_{\omega}=0.5 \quad ; \quad \sigma_{k}=2 / 3 \quad ; \quad \sigma_{d}=0.5
$$

The constant values were changed, following all constraints, to show the sensitivity of the model to the cross-diffusion term $\operatorname{grad} k \cdot \operatorname{grad} \omega$ in the $\omega$ equation for these unsteady computations.

$$
\begin{array}{ll}
\text { test } 1: & \sigma_{\omega}=0.5 ; \sigma_{k}=2 / 3 ; \sigma_{d}=0.65 \\
\text { test 2: } & \sigma_{\omega}=0.5 ; \sigma_{k}=1 ; \sigma_{d}=0.85
\end{array}
$$

\subsection{Wall law approach}

At the wall, a no-slip condition was used coupled to a wall law treatment. It consists in imposing the diffusive flux densities, required for the integration process, in adjacent cells to a wall. The shear stress $\tau$ and the heat flux $q$ are obtained from an analytical velocity profile :

$$
\begin{array}{llll}
\bar{u}^{+} & =y^{+} & \text {if } & y^{+}<11.13 \\
\bar{u}^{+}=\frac{1}{\kappa} \ln y^{+}+5.25 & \text { if } & y^{+}>11.13 \\
\bar{u}^{+}=\bar{u} / U_{\tau} & & ; & y^{+}=\frac{y U_{\tau}}{\nu_{w}}
\end{array}
$$

In equation (9), $\bar{u}$ represents the van Driest $[36,37]$ transformed velocity for compressible flows. 
Concerning transport-equation turbulence models, $k$ was set to 0 at the wall and its production was imposed according to the formulation proposed by Viegas and Rubesin [38, 39]. The second variable was deduced from an analytical relation and was imposed in adjacent cells to a wall. The characteristic length scale of the Chen model [40] was used for the dissipation rate $\varepsilon$ and the specific dissipation $\omega$. For the Smith model, a standard linear law for the length $l$ was used.

For the one-equation Spalart-Allmaras model, the transported quantity was imposed in adjacent cells to a wall by using the closure relations of the model, the velocity profile and a mixing-length formulation for the eddy-viscosity. More details concerning the wall law approach are given in [11].

For unsteady boundary layers, the existence of a wall law was assumed valid at each instant. As shown in [41], the velocity phase shift is nearly constant in the logarithmic region and equal to the shift of the wall shear stress phase. This is true for a Strouhal number up to 10 .

When using the wall law approach with the multigrid algorithm, the wall law boundary condition was applied on the fine grid and the no-slip condition was applied on the coarse grids.

\section{$3 \quad$ Numerical results}

\subsection{Experimental conditions}

The experimental study was conducted in the S3MA ONERA wind tunnel [42] with the RA16SC1 airfoil. It is a supercritical airfoil with a relative thickness equal to $16 \%$ and a chord length equal to $180 \mathrm{~mm}$. The RMS pressure fluctuations were measured from 36 Kulite transducers installed in the airfoil. The flow conditions were : $M_{\infty}=0.732$, $T_{i}=283 K, R e_{c}=4.210^{6}$ and the angle of attack varied from 0 to $4.5^{\circ}$. Transition was fixed near the leading edge at $x / c=7.5 \%$ on both sides of the airfoil. 


\subsection{Computational conditions}

For the computations, experimental corrections were used. The Mach number was decreased by 0.09 and the angle of attack was decreased by $1^{\circ}$ at all incidences with respect to experiment. The grid was a C-type topology. It contained $321 \times 81$ nodes, 241 of which were on the airfoil(cf. figure 1,2$)$. The $y^{+}$values of the coarse mesh, at the center of the first cell, are presented in figure 3 for a steady computation at $\alpha=4^{\circ}$.

The numerical parameters used for the computations were :

- the dimensionless time step, $\Delta t^{*}=\frac{\Delta t a_{i}}{c}=0.2$

where $c$ is the chord of the airfoil and $a_{i}$ the stagnation sound velocity

- grid levels for the multigrid method, 2

- sub-iterations of the dual time stepping method, 75 up to 100

By increasing the number of sub-iterations, it was checked that the same solution was achieved.

- the CFL number, 200

- Jacobi iterations for the implicit stage, 14

- the artificial dissipation of the Jameson scheme introduces two coefficients, one for the second-difference term: $\chi_{2}=0.5$ and one for the fourth-difference term: $\chi_{4}=0.016$. For the second grid level, the coefficient $\chi_{4}$ was fixed at 0.032

- the coefficient of the Harten's correction, 0.05

Computations started from a uniform flow-field using a local time step and one grid level. After 50 iterations, the dual time stepping method was used with the mulgrid algorithm and oscillations develop with a growing amplitude.

\subsection{Comparison of turbulence models}

The frequency $f$ and the amplitude of the lift coefficient $\Delta C_{L}$ are reported in table 1 for all turbulence models and for three angles of attack $\alpha=3,4$ and $5^{\circ}$, corresponding to the buffet onset, established phenomenon and buffet exit, i.e. the return to a steady state, respectively.

The capacity of turbulence models to restitute the natural unsteadiness of the flow 
without and with any correction was first examinated.

The Spalart-Allmaras model can reproduce the buffet phenomenon, the frequency being underestimated with respect to the experimental values. The lift amplitude was very weak for the buffet onset and the buffet exit was not obtained.

The Smith $k-l$ model needs a correction to obtain unsteady results. The Smith correction does not seem to be efficient for these unsteady computations. Yet, the SST corrections enable the model to simulate the buffet. As for the Spalart-Allmaras model, the lift amplitude was largely underestimated for the buffet onset and the buffet exit was not predicted. The Jones-Launder $k-\varepsilon$ model can provide unsteady solutions without any correction. Yet, the lift amplitude was largely underestimated for $\alpha=4^{\circ}$ and the model completely damped the natural unsteadiness for the onset. The shock-induced oscillations appear at an angle of attack of $3.7^{\circ}$ rather than $3^{\circ}$ for the experimental value. Thanks to the addition of the SST correction a larger amplitude of the lift coefficient was obtained but the buffet onset was not predicted. The realizability conditions of Durbin enable the model to predict the buffet onset but the lift coefficient amplitude obtained is largely underpredicted. For the established phenomenon, the amplitude is closer to the experimental value when using the Durbin correction in comparison with the use of the SST correction. The back to a steady state was not simulated for the three computations with the $k-\varepsilon$ model.

The Wilcox and Menter $k-\omega$ models fail to compute this application, the results obtained being completely steady. Adding the cross-diffusion term $\operatorname{grad} k \cdot \operatorname{grad} \omega$ in the $\omega$ equation of the Menter model, in comparison with the Wilcox model, does not enable the model to predict shock-induced oscillations. Adding the SST correction to the Menter model has a great influence and allows self-sustained oscillations to be predicted with a very good agreement with respect to the experimental data.

The Kok $k-\omega$ model can compute natural unsteadiness for the established phenomenon but the buffet onset and the buffet exit are not predicted. It seems that the SST corrections and the realizability constraints do not modify the behaviour of the model.

The recalibration of the constant of the Kok model was tested for the three angles of attack. The frequency and amplitude of the lift coefficient are reported in table 2. Increasing the $\sigma_{d}$ coefficient induced an increased amplitude of the lift coefficient for all angles of attack and allowed the prediction of the entrance in the SIO domain. Yet, there is no buffet exit at $\alpha=5^{\circ}$. 
When comparing all turbulence models, the best results are clearly obtained with the SST Menter model, for the three angles of attack. The amplitude of the lift coefficient is remarkably predicted and the buffet exit is only predicted when using this model. All these results show the interest of the use of a correction for this unsteady application.

The RMS values of the pressure fluctuations over the airfoil are compared in figure 4 with experimental results at the angle of attack $\alpha=3^{\circ}$. The pressure side is represented by the negative values of the abscisse. The SST Menter model clearly provides the best result. Over the pressure side the computed pressure fluctuation is in very close agreement with the measured values. The peak on the upper side, corresponding to the shock movement, is well located but underestimated by $15 \%$. The results obtained by the other turbulence models are very far from the experimental data, pressure fluctuations over the airfoil being largely underestimated.

Figure 5 presents the RMS pressure fluctuations over the airfoil obtained with the modified Kok $k-\omega$ models. For the two tests, the peak over the upper side is at a downstream location in comparison with the experiment. Both models under-estimate the maximum value on the upper side, especially the test- 1 modified model and the amplitude of the shock displacement. Over the pressure side, the test-1 Kok model under-predicts the level of pressure fluctuations. It seems that the increase in the coefficient $\sigma_{d}$ allows a better capture of the unsteadiness of the flow.

The RMS values of the pressure fluctuations over the airfoil are plotted in figure 6 for an angle of attack $\alpha=4^{o}$ and just for the $k-\varepsilon$ models. The great influence of the SST correction and the realizability constraints can be observed. Without any correction, the pressure fluctuations are largely under-estimated on the pressure side and on the trailing edge of the upper side. The amplitude of the shock displacement are too weak in comparison with the experimental values and the peak is not well located on the upper side. With corrections, the pressure fluctuations on the trailing edge of the upper side are close to the experimental data. The amplitude of the shock and the peak location are in better agreement with the experiment. Yet, the maximum value on the upper side is over-predicted while, on the pressure side, the fluctuations level is over-estimated. The change of the 
value of the constant $c$ in the realizability constraints should improve the results.

The RMS pressure fluctuations over the airfoil obtained with the Kok $k-\omega$ models are the same and are not plotted together. In figure 7, the RMS pressure fluctuations are plotted for all generic turbulence models. Over the pressure side, the $k-\varepsilon$ model with the Durbin correction over-predicts the pressure fluctuation and all other models give good results. Over the upper side, the peak is well located except for the Kok model. The maximum value is under-estimated by the SST Menter model. Downstream the shock location, at the trailing edge, a large discrepancy with experimental values, which can reach $50 \%$, is observed for all models.

Finally, the RMS values of the pressure fluctuations over the airfoil are presented in figure 8 for the Kok $k-\omega$ models. When the constant $\sigma_{d}$ is increased, the displacement of the shock over the upper side is extended and the pressure levels become more important.

\subsection{Influence of the numerical scheme}

The previous paragraph analyzed various turbulence models computed with one numerical scheme for the mean flow : the Jameson scheme. This part presents the influence of the numerical scheme, all computations being carried out with one turbulence model. For the quality of results, the SST Menter model was selected. Concerning the integration of the turbulent transport equations, a second-order Roe scheme is always used.

We consider the upwind Roe scheme [15], the AUSM + Liou scheme [43] and the Jameson scheme in which the dispersive error is cancelled. The Roe and Liou schemes being of first-order spatial accuracy, the MUSCL extrapolation is used to increase the spatial accuracy. Extrapolated values at a cell interface are given by :

$$
\begin{aligned}
& w_{i+1 / 2, j}^{L}=w_{i, j}+\frac{1}{4}\left[(1-\kappa)\left(w_{i, j}-w_{i-1, j}\right)+(1+\kappa)\left(w_{i+1, j}-w_{i, j}\right)\right] \\
& w_{i-1 / 2, j}^{R}=w_{i, j}-\frac{1}{4}\left[(1+\kappa)\left(w_{i, j}-w_{i-1, j}\right)+(1-\kappa)\left(w_{i+1, j}-w_{i, j}\right)\right]
\end{aligned}
$$

The constant $\kappa$ is set to $1 / 3$. This choice allows the dispersive error to be minimized and the third-order spatial accuracy to be approached. Although the flow presents a disconti- 
nuity with the shock wave, no slope limiter, ensuring the TVD property, was used. Indeed, computations of the buffet over transonic airfoil with a limited Roe-MUSCL scheme showed the great influence of the slope limiter on the amplitude of the lift coefficient [44].

The frequency and amplitude of the lift coefficient are reported in table 3 for all numerical schemes and for the three angles of attack. For the entrance in the SIO domain, at $\alpha=3^{\circ}$, the amplitude of the lift coefficient is largely under-estimated with the Liou and Roe schemes. In comparison with the experiment, the lift amplitude obtained with the Jameson corrected is less close to the result obtained with the Jameson scheme.

For the established phenomenon, at $\alpha=4^{\circ}$, the Liou and Roe schemes gave very close results with respect to the experiment, in comparison with the result obtained with the Jameson scheme. The use of the Jameson corrected scheme allows the improvement of the frequency and amplitude of the lift coefficient.

For the buffet exit, at $\alpha=5^{\circ}$, the back to a steady state is predicted by all schemes except by the Jameson corrected one. The computed exit of the SIO domain is probably due to a numerical artefact.

The RMS values of the pressure fluctuations over the airfoil are plotted in figure 9 for an angle of attack $\alpha=4^{\circ}$. The Jameson schemes provide approximately the same solution. The Roe and Liou schemes largely under-predict the peak in the suction side. It is surprising that the Jameson scheme gives better results than the Roe and Liou schemes which are less dissipative.

To explain these surprising results, weighted schemes were implemented to take into consideration the mesh deformation. Indeed, as shown in figure 2, the second adjacent cell to a wall is largely finer than the first one, due to the use of a wall law approach. This important change of cell size induces a loss of spatial accuracy which can be corrected. The centered numerical fluxes and the gradient computations are corrected by using a weighted discretization operator $\tilde{\mu} w_{i+1 / 2}$ instead of the classical operator $\mu w_{i+1 / 2}=0.5\left(w_{i+1}+w_{i}\right)$. Let $A$ and $B$ two points and $M$ an interior point of the segment $A B$, the weighted discrete operator is defined by :

$$
\tilde{\mu}_{A}^{B} w_{M}=\frac{M B}{A B} w_{B}+\frac{A M}{A B} w_{A}
$$


For the MUSCL reconstruction, a correction is also used, the extrapolated values at a cell interface become, for three points $P, Q, R$ corresponding to $i-1, i, i+1$ :

$$
\begin{aligned}
& w_{i+1 / 2, j}^{L}=w_{i, j}+\frac{P R}{2 P Q}\left[(1-\kappa)\left(w_{i, j}-w_{i-1, j}\right)+\left(1+\frac{P R}{R Q} \kappa\right)\left(w_{i+1, j}-w_{i, j}\right)\right] \\
& w_{i-1 / 2, j}^{R}=w_{i, j}-\frac{P R}{2 P Q}\left[(1+\kappa)\left(w_{i, j}-w_{i-1, j}\right)+\left(1-\frac{P R}{R Q} \kappa\right)\left(w_{i+1, j}-w_{i, j}\right)\right]
\end{aligned}
$$

It is also possible to take into account the mesh deformation in the computation of the Jameson artificial dissipation. The formulation of the third derivative of the conservative variable $\delta^{3} w$, for four points $M, P, Q, R$ corresponding to $i-2, i-1, i, i+1$, can be expressed :

$\delta^{3} w_{i+1 / 2, j}=6 P Q^{3}\left(\frac{w_{i+1, j}}{R Q \times P R \times M R}+\frac{w_{i, j}}{Q R \times P Q \times M Q}+\frac{w_{i-1, j}}{P Q \times P R \times M P}-\frac{w_{i-2, j}}{M Q \times M R \times P M}\right)$

Yet, the use of the corrected artificial dissipation (called Jameson weighted 2 in the following) makes the convergence more difficult to obtain. The $\chi_{4}$ coefficient is set to 0.032 for the first level grid. The Jameson scheme in which the dispersive error has been cancelled is not tested with a weighted formulation.

The frequency and the amplitude of the lift coefficient are reported on the table 4 only for one angle of attack $\alpha=4^{\circ}$. The weighted correction yields an increase of the amplitude of the lift coefficient and a reduction of the frequency for all schemes.

The RMS values of the pressure fluctuations over the airfoil are plotted in figure 10 for $\alpha=4^{o}$. We can see that the weighted correction allows to improve the result for all schemes in comparison with the experimental values and with the standard Jameson scheme. Moreover, results obtained with the Roe and Liou schemes are approximatively identical and are very closer to those obtained with the Jameson weighted scheme. We choose to use the Roe-MUSCL weighted scheme in the following of the article rather than the Jameson weighted scheme. Indeed, it allows to eliminate two parameters, the $\chi_{2}$ and $\chi_{4}$ coefficients.

\subsection{Influence of the velocity profile in the wall law boundary condition}

The wall law approach is based on the use of an analytical velocity profile in the turbulent boundary layer. The two-layer model composed by a linear law and a logarithmic law is 
one of the simplest. We have implemented the Spalding law [45], more sophisticated, to evaluate the influence of the velocity profile. This law is given by :

$$
y^{+}=u^{+}+\exp (-\kappa C)\left[\exp \left(\kappa u^{+}\right)-1-\kappa u^{+}-\frac{\left(\kappa u^{+}\right)^{2}}{2}-\frac{\left(\kappa u^{+}\right)^{3}}{6}\right]
$$

The wall skin friction is computed from this law with a Newton algorithm .

A computation is realized with the Roe-MUSCL weighted scheme and the SST Menter turbulence model for an angle of attack $\alpha=4^{\circ}$. The frequency and the amplitude of the lift coefficient are reported on the table 5 .

We note that the frequency of the shock induced oscillations and the amplitude of the lift coefficient are identical with the two formulations.

The RMS values of the pressure fluctuations over the airfoil are plotted in figure 11 . Results are very close, a little discrepancy is observed on the peak on the upper side. It seems that the velocity profile has a weak influence for these unsteady computations.

\subsection{Influence of the Harten's entropy correction}

For the turbulence transport equation integration, numerical fluxes are computed with a second order Roe scheme in which the Harten correction is added. This correction was used for transonic applications and improved the robustness of computations integrated down to a wall with a very fine mesh. The correction acts on the eigenvalue of the turbulent system, the normal velocity to an interface, by truncating it near the wall. There is, a priori, no solid arguments to use it for the turbulent system except the convergence aspect.

With the use of a wall law treatment, the robustness is largely improved and the Harten correction can be cancelled. A computation is realized with the Roe-MUSCL weighted scheme, the SST Menter turbulence model, the Spalding velocity profile and without any Harten correction for an angle of attack $\alpha=4^{\circ}$. The frequency and the amplitude of the lift coefficient are reported on the table 6 . We can see that the Harten correction has a significant influence on the amplitude of the lift coefficient and limits the buffet phenomenon. 
The RMS values of the pressure fluctuations over the airfoil are plotted in figure 12 . The Harten correction decreases the displacement of the shock wave over the upper side and limits the pressure fluctuations over the airfoil. Without the Harten correction, results obtained are in better agreement with the experiment over the suction side and over-predict the pressure fluctuations over the pressure side.

\subsection{Simulation of the buffet}

Computations are made with the SST Menter turbulence model, the Roe-MUSCL weighted scheme, the Spalding velocity profile and without the Harten correction for seven angles of attack from $\alpha=3^{\circ}$ up to $\alpha=6^{\circ}$.

The entrance in the SIO domain is well predicted by the numerical simulation for both frequency and amplitude of the phenomenon. The back to a steady state is evidenced by the computations for an angle of attack of $6^{\circ}$. That is one degree more in comparison with the experimental value. It clearly shows the influence of the numerics on theses unsteady computations for the buffet exit.

The evolution of the reduced frequency $2 \pi f c / U_{\infty}$ and the RMS amplitude of the lift coefficient versus the angle of attack are plotted in figures 13 and 14. Discrepancies between computations and experimental data are observed but the tendency is well reproduced by the computations. As the angle of attack grows, the frequency of SIO increases, the amplitude of the phenomenon reaches a maximum value and decreases up to zero. Unfortunately, the computed frequency is under-estimated and the RMS amplitude is over-predicted. It is difficult to explain the gap of one degree for the exit of the SIO domain.

The RMS values of the pressure fluctuations over the airfoil are plotted in figures 15, 16 and 17 for three angles of attack. The evolution of pressure fluctuations is remarkably estimated over the both side for all computations. The peak value is a little under-estimated for the entrance of the buffet. For the other angle of attack, the peak and the amplitude of the shock displacement are well computed. Over the trailing edge of the pressure side, RMS values are in good agreement with the experiment, the largest discrepancies are observed at the buffet onset. 


\section{Conclusion}

The unsteady two-dimensional computations of the transonic buffet over a supercritical airfoil are performed with an implicit solver which reveals the great sensitivity to the turbulence modeling and the numerical schemes. Usual turbulence models fail in correctly predicting SIO and the introduction of a weakly non-linear correction in the definition of the eddy viscosity yields better results. Two different approaches are tested, the use of the empirical Bradshaw's assumption through the SST correction and the enforcement of the realizability principle. Another approach consists in recalibrating the constant of the model for unsteady flows. For the Kok model, by increasing the constant of the crossdiffusion term, results are improved and the buffet onset can be predicted.

The paper presents also the influence of the numerical schemes and the significant improvments brought by considering the mesh deformation especially for the Roe and AUSM + Liou schemes. The numerics has also a significant influence for the computation of the SIO domain exit to a steady state.

Finally, the complete SIO domain is computed with a weakly non-linear turbulence model and a weighted scheme associated with a wall law approach for the RA16SC1 airfoil. The evolution of the frequency and the amplitude of the phenomenon is qualitatively well predicted. The buffet exit is also well reproduced but the corresponding angle of attack is shifted by one degree. Yet, the RMS pressure fluctuations over the airfoil, directly relies on the physics of the phenomenon, are in very good agreement with experimental values. 


\section{References}

[1] Tijdeman H. Investigation of the transonic flow around oscillating airfoils. NLR, TR77-090U. 1977.

[2] Lee BHK. Self-Sustained Shock Oscillations on airfoils at transonic speeds. Progress in Aerospace Sciences. 2001; 37: 147-196.

[3] Renaud T, Corre C, Lerat A. Efficient Numerical Simulation of Buffet for Airfoils in Transonic Regime. Proceedings of IFASD 2001, Madrid, Spain, 5-7 June.

[4] Cinnella P, Lerat A. A fully implicit third-order scheme in time and space for compressible turbulent unsteady flow simulations. Proceedings of ECCOMAS 2000, Barcelona, Spain, 11-14 September.

[5] Ekaterinaris JA, Menter FR. Computation of oscillating airfoil flows with one and two equation turbulence models. AIAA Journal. 1994; 32: 2359-2365.

[6] Furlano F, Goncalves E, Houdeville R, Coustols E. Unsteady RANS Computations : Simulation of the Buffeting over an Airfoil. Proceedings of the IUTAM Symposium on Unsteady Separated Flows, Toulouse, France, April 8-12, 2002.

[7] Srinivasan GR, Ekaterinaris JA, McCroskey J. Evaluation of turbulence models for unsteady flows of an oscillating airfoil, Computers \& Fluids. 1995; 7: 833-861.

[8] Wang D, Wallin S, Berggren M, Eliasson P. A computational study of unsteady turbulent buffet aerodynamics. AIAA Paper 2000-2657, Denver, Colorado, 2000.

[9] Barakos G, Drikakis D. Numerical Simulation of Transonic Buffet Flows Using Various Turbulence Closures. International Journal of Heat and Fluid Flow. 2000; 21: 620-626.

[10] Goncalves E, Houdeville R. Numerical simulation of shock oscillations over airfoil using a wall law approach. AIAA Journal. 2003; 41: 1829-1832.

[11] Goncalves E, Houdeville R. Reassessment of the wall functions approach for RANS computations. Aerospace Science and Technology. 2001; 5: 1-14.

[12] Ha Minh H. Order and disorder in turbulent flows : their impact on turbulence modelling. Osborne Reynolds centenary symposium,Manchester, 24 May, 1994. 
[13] Jin G, Braza M. Two-equation turbulence model for unsteady separated flows around airfoils. AIAA Journal. 1994; 32: 2316-2320.

[14] Jameson A, Schmidt W, Turkel E. Numerical simulation of the Euler equations by finite volume method using Runge-Kutta time stepping schemes. AIAA Paper 81-1259, 1981.

[15] Roe PL. Approximate Riemann solvers, parameters vectors, and difference schemes. Journal of Computational Physics. 1981; 43; 357-372.

[16] Tatsumi S, Martinelli L, Jameson A. Flux-Limited Schemes for the Compressible Navier-Stokes Equations. AIAA Journal. 1995; 33; 252-261.

[17] Luo H, Baum JD, Lohner R. A fast, matrix-free implicit method for compressible flows on unstructured grids. Journal of Computational Physics. 1998; 146: 664-690.

[18] Shende N, Arora K, Balakrishnan N. Convergence acceleration using implicit relaxation procedures for cell centre and cell vertex finite volume schemes. Fluid Mechanics Report, No. 2001 FM 03, Indian Institute of Science, 2001.

[19] Merci B, Steelant J, Vierendeels J, Riemslagh K, Dick E. Computational treatment of source terms in two-equation turbulence models. AIAA Journal. 2000; 38; 2085-2093.

[20] Jameson A. Multigrid Algoritms for Compressible Flow Calculations. In Lecture Notes in Mathematics $n^{\circ}$ 1228, Springer-Verlag, 1985.

[21] Jameson A. Time Dependent Calculations Using Mutligrid with Applications to Unsteady Flows past Airfoils and Wings. AIAA Paper 91-1259, Honolulu, Hawai, 1991.

[22] Thomas JL, Salas MD. Far-Field Boundary Conditions for Transonic Lifting Solutions to the Euler Equations. AIAA Journal. 1986; 24: 1074-1080.

[23] Smith BR. The $k-k l$ Turbulence Model and Wall Layer Model for Compressible Flows. AIAA Paper 90-1483, Seattle, Washington, 1990.

[24] Smith BR. A Near Wall Model for the $k-l$ Two Equation Turbulence Model. AIAA Paper 94-2386. Colorado Springs, Colorado, June 20-23, 1994.

[25] Wilcox DC. Reassessment of the scale-determining equation for advanced turbulence models. AIAA Journal. 1988; 26: 1299-1310. 
[26] Menter FR. Zonal two equation $k-\omega$ turbulence models for aerodynamic flows. AIAA 93-2906, 24 th Fluid Dynamics Conference, Orlando, Florida, July 6-9 1993.

[27] Menter FR. Two-equation eddy-viscosity turbulence models for engineering applications. AIAA Journal. 1994; 32: 1598-1605.

[28] Jones WP, Launder BE. The Prediction of Laminarization with a Two-Equation Model of Turbulence. International Journal of Heat and Mass Transfer. 1972; 15: 301-314.

[29] Kok JC. Resolving the Dependence on Freestream Values for the $k-\omega$ turbulence model. AIAA Journal. 2000; 38: 1292-1295.

[30] Spalart PR, Allmaras SR. A one-equation turbulence model for aerodynamic flows. AIAA Paper 92-0439, Reno, Nevada, 6-9 January, 1992.

[31] Spalart PR, Allmaras SR. A one-equation turbulence model for aerodynamic flows. La Recherche Aerospatiale. 1994; 1: 5-21.

[32] Smith BR. A nonequilibrium Turbulent Viscosity Function for the $k-l$ Two Equation Turbulence Model. AIAA Paper 97-1959, Snowmass Village, Colorado, 1997.

[33] Durbin PA. On the $k-3$ stagnation point anomaly. International Journal of Heat and Fluid Flow. 1996; 17: 89-90.

[34] Behnia A., Parneix S, Durbin PA. Simulation of jet impingement heat transfer with the $k-\varepsilon-v^{2}$ model. Annual Research Briefs, Standford University, Center for Turbulence Research, december 1996.

[35] Thivet F. Lessons Learned from RANS Simulations of Shock-Wave/Boundary Layer Interactions. AIAA Paper 2002-0583, Reno, Nevada, January 14-17, 2002.

[36] van Driest ER. Turbulent Boundary Layer in Compressible Fluids. J. Aeronaut. Sci., 1951; 18: 145-160.

[37] van Driest ER. On Turbulent Flow Near a Wall. J. Aeronaut. Sci.. 1957; 23: 10071011.

[38] Viegas JR, Rubesin MW. Wall-Function Boundary Conditions in the Solution of The Navier-Stokes Equations for Complex Compressible Flows. AIAA Paper 83-1694, Danver, Massachussetts, 12-14 July, 1983. 
[39] Viegas JR, Rubesin MW, Horstman CC. On the Use of Wall Functions as Boundary Conditions for Two-Dimensionnal Separated Compressible Flows. AIA A Paper 85-0180, Reno, Nevada, 14-17 January, 1985.

[40] Chen HC, Patel VC. Near-Wall Turbulence Models for Complex Flows Including Separation. AIAA Journal. 1985; 26: 641-648.

[41] Cousteix J, Houdeville R, Javelle J. Response of a turbulent boundary layer to a pulsation of the external flow with and without adverse pressure gradient. Proceedings of the International Union of Theoretical and Applied Mechanics Symposium, Toulouse, France, 5-8 May, 1981.

[42] Benoit B, Legrain I. Buffeting Prediction for Transport Aircraft Applications Based on Unsteady Pressure Measurements. AIAA Paper 87-2356, Montery, California, 1987.

[43] Liou MS. Ten years in the making AUSM-family. AIAA Paper 2001-2521, Anaheim, California, 11-14 June, 2001.

[44] Renaud T. Simulation numerique du tremblement autour de profils d'ailes en regime transsonique. Ph.D. Thesis. ENSAM-Paris, France, 2001.

[45] Spalding DB. A single formula for the law of the wall. Journal of Applied Mechanics. 1961 ; bf $17(5)$. 


\begin{tabular}{||c||c|c||c|c||c|c||}
\hline \multicolumn{1}{||c||}{} & \multicolumn{2}{c||}{$\alpha=3^{\circ}$} & \multicolumn{2}{c||}{$\alpha=4^{\circ}$} & \multicolumn{2}{c||}{$\alpha=5^{\circ}$} \\
\hline model & $\mathrm{f}(\mathrm{Hz})$ & $\Delta C_{L}$ & $\mathrm{f}(\mathrm{Hz})$ & $\Delta C_{L}$ & $\mathrm{f}(\mathrm{Hz})$ & $\Delta C_{L}$ \\
\hline \hline experiment & $\mathbf{8 8}$ & $\mathbf{0 . 1 1}$ & $\mathbf{1 0 0}$ & $\mathbf{0 . 3 0 8}$ & \multicolumn{2}{|c||}{$\begin{array}{c}\text { probably } \\
\text { steady state }\end{array}$} \\
\hline \hline Spalart-Allmaras & 82 & 0.0146 & 92 & 0.325 & 100 & 0.55 \\
\hline \hline$k-l$ & - & - & steady state & - & - \\
\hline$k-l$ corrected & - & - & steady state & - & - \\
\hline$k-l$ SST & 79.5 & 0.0084 & 97.6 & 0.296 & 101.8 & 0.53 \\
\hline \hline$k-\varepsilon$ & steady state & 95.6 & 0.17 & 97.6 & 0.43 \\
\hline$k-\varepsilon$ SST & steady state & 95.6 & 0.48 & 101.8 & 0.67 \\
\hline \hline$k-\omega$ Wirbin & 85.2 & 0.012 & 93.7 & 0.437 & 101.8 & 0.67 \\
\hline$k-\omega$ Menter & - & - & steady state & - & - \\
\hline$k-\omega$ SST Menter & 90 & 0.11 & 96.6 & 0.33 & steady state \\
\hline \hline$k-\omega$ Kok & \multicolumn{2}{|c||}{ steady state } & 94.6 & 0.26 & 95.6 & 0.48 \\
\hline$k-\omega$ Kok SST & \multicolumn{2}{|c||}{ steady state } & 94.6 & 0.26 & 96.6 & 0.445 \\
\hline$k-\omega$ Kok Durbin & steady state & 94.6 & 0.26 & 96.6 & 0.45 \\
\hline
\end{tabular}

Table 1: Frequency and amplitude of the lift coefficient 


\begin{tabular}{||c||c|c||c|c||c|c||}
\hline \multicolumn{1}{||c||}{} & \multicolumn{2}{c||}{$\alpha=3^{\circ}$} & \multicolumn{2}{c||}{$\alpha=4^{\circ}$} & \multicolumn{2}{c||}{$\alpha=5^{\circ}$} \\
\hline model & $\mathrm{f}(\mathrm{Hz})$ & $\Delta C_{L}$ & $\mathrm{f}(\mathrm{Hz})$ & $\Delta C_{L}$ & $\mathrm{f}(\mathrm{Hz})$ & $\Delta C_{L}$ \\
\hline \hline experiment & $\mathbf{8 8}$ & $\mathbf{0 . 1 1}$ & $\mathbf{1 0 0}$ & $\mathbf{0 . 3 0 8}$ & \multicolumn{2}{|c||}{ steady state } \\
\hline$k-\omega$ Kok & \multicolumn{2}{||}{ steady state } & 94.6 & 0.26 & 95.6 & 0.48 \\
\hline$k-\omega$ Kok - test 1 & 91 & 0.051 & 93.7 & 0.318 & 95.6 & 0.55 \\
\hline$k-\omega$ Kok - test 2 & 87.6 & 0.084 & 91 & 0.46 & 91 & 0.735 \\
\hline
\end{tabular}

Table 2: Frequency and amplitude of the lift coefficient - Kok model 


\begin{tabular}{||c||c|c||c|c||c|c||}
\hline \multicolumn{1}{||c||}{} & \multicolumn{2}{c||}{$\alpha=3^{\circ}$} & \multicolumn{2}{c||}{$\alpha=4^{\circ}$} & \multicolumn{2}{c||}{$\alpha=5^{\circ}$} \\
\hline model & $\mathrm{f}(\mathrm{Hz})$ & $\Delta C_{L}$ & $\mathrm{f}(\mathrm{Hz})$ & $\Delta C_{L}$ & $\mathrm{f} \mathrm{(Hz)}$ & $\Delta C_{L}$ \\
\hline \hline experiment & $\mathbf{8 8}$ & $\mathbf{0 . 1 1}$ & $\mathbf{1 0 0}$ & $\mathbf{0 . 3 0 8}$ & \multicolumn{2}{|c||}{$\begin{array}{c}\text { probably } \\
\text { steady state }\end{array}$} \\
\hline Jameson & 90 & 0.11 & 96.6 & 0.33 & \multicolumn{2}{|c||}{ steady state } \\
\hline Roe MUSCL & 90 & 0.014 & 99.7 & 0.3 & \multicolumn{2}{|c||}{ steady state } \\
\hline AUSM+ MUSCL & 90 & 0.018 & 98.6 & 0.307 & \multicolumn{2}{|c||}{ steady state } \\
\hline Jameson corrected & 91 & 0.097 & 97.6 & 0.327 & 99.7 & 0.46 \\
\hline
\end{tabular}

Table 3: Frequency and amplitude of the lift coefficient 


\begin{tabular}{||c||c|c||}
\hline \multicolumn{1}{||c||}{} & \multicolumn{2}{c||}{$\alpha=4^{\circ}$} \\
\hline scheme & $\mathrm{f}(\mathrm{Hz})$ & $\Delta C_{L}$ \\
\hline \hline experiment & $\mathbf{1 0 0}$ & $\mathbf{0 . 3 0 8}$ \\
\hline Jameson & 96.6 & 0.33 \\
\hline Jameson weighted & 96.6 & 0.346 \\
\hline Jameson weighted 2 & 95.6 & 0.343 \\
\hline Roe MUSCL & 99.7 & 0.30 \\
\hline Roe MUSCL weighted & 96.6 & 0.34 \\
\hline AUSM+ MUSCL & 98.6 & 0.307 \\
\hline AUSM+ MUSCL weighted & 98.6 & 0.358 \\
\hline
\end{tabular}

Table 4: Frequency and amplitude of the lift coefficient 


\begin{tabular}{||c||c|c||}
\hline \multicolumn{1}{||c||}{} & \multicolumn{2}{c||}{$\alpha=4^{\circ}$} \\
\hline velocity profile & $\mathrm{f}(\mathrm{Hz})$ & $\Delta C_{L}$ \\
\hline \hline experiment & $\mathbf{1 0 0}$ & $\mathbf{0 . 3 0 8}$ \\
\hline two-layer model & 96.6 & 0.34 \\
\hline Spalding law & 96.6 & 0.34 \\
\hline
\end{tabular}

Table 5: Frequency and amplitude of the lift coefficient 


\begin{tabular}{||c||c|c||}
\hline \multicolumn{1}{||c||}{} & \multicolumn{2}{c||}{$\alpha=4^{\circ}$} \\
\hline & $\mathrm{f}(\mathrm{Hz})$ & $\Delta C_{L}$ \\
\hline \hline experiment & $\mathbf{1 0 0}$ & $\mathbf{0 . 3 0 8}$ \\
\hline with Harten correction & 96.6 & 0.34 \\
\hline without Harten correction & 93.7 & 0.40 \\
\hline
\end{tabular}

Table 6: Frequency and amplitude of the lift coefficient 


\begin{tabular}{||c||c|c||c|c||}
\hline \multicolumn{1}{||c||}{} & \multicolumn{2}{c||}{ SST Menter model } & \multicolumn{2}{c||}{ experiment } \\
\hline angle of attack & $\mathrm{f}(\mathrm{Hz})$ & $\Delta C_{L}$ & $\mathrm{f}(\mathrm{Hz})$ & $\Delta C_{L}$ \\
\hline \hline$\alpha=3^{\circ}$ & 89 & 0.106 & 88 & 0.11 \\
\hline$\alpha=3.5^{\circ}$ & 90.1 & 0.28 & 92 & 0.25 \\
\hline$\alpha=4^{\circ}$ & 93.7 & 0.40 & 100 & 0.31 \\
\hline$\alpha=4.5^{\circ}$ & 96.6 & 0.44 & 108 & 0.26 \\
\hline$\alpha=5^{\circ}$ & 98.6 & 0.50 & probably steady state \\
\hline$\alpha=5.5^{\circ}$ & 104 & 0.47 & \multicolumn{2}{c||}{-} \\
\hline$\alpha=6^{\circ}$ & \multicolumn{3}{|c||}{ steady state } & \multicolumn{2}{c||}{} \\
\hline
\end{tabular}

Table 7: Frequency and amplitude of the lift coefficient 


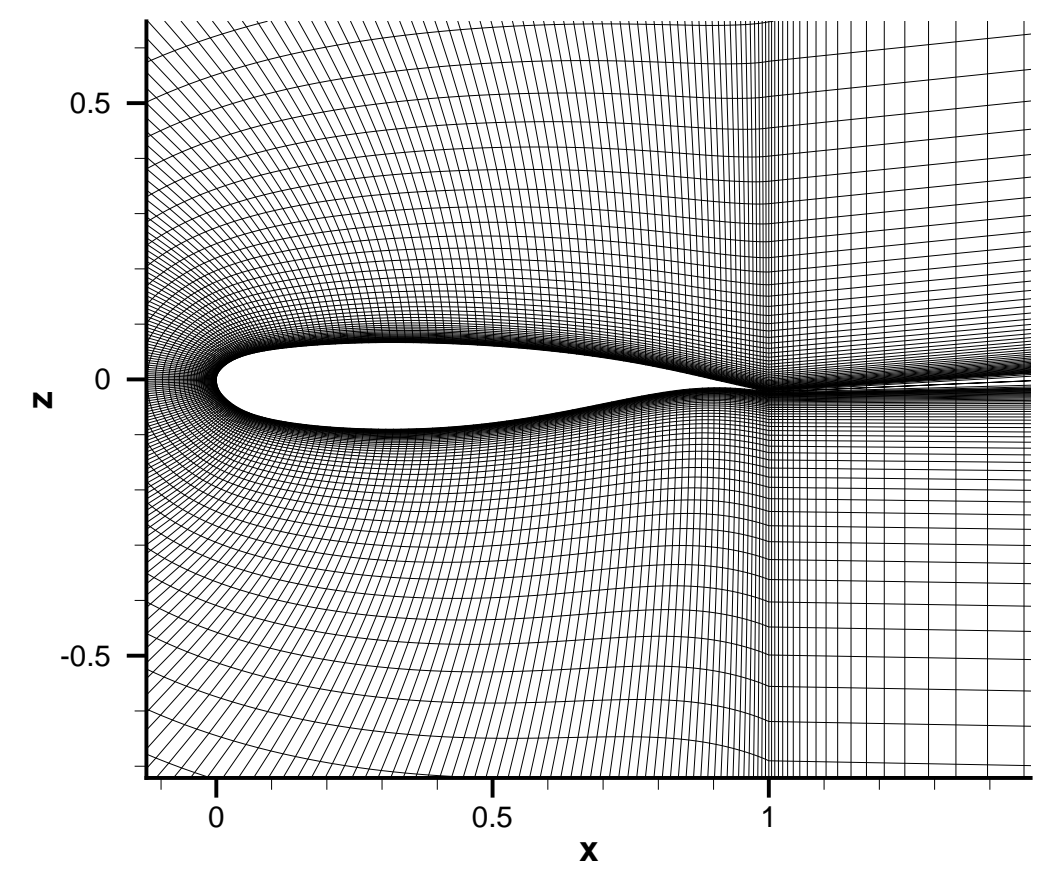

Figure 1: Mesh 321x81 


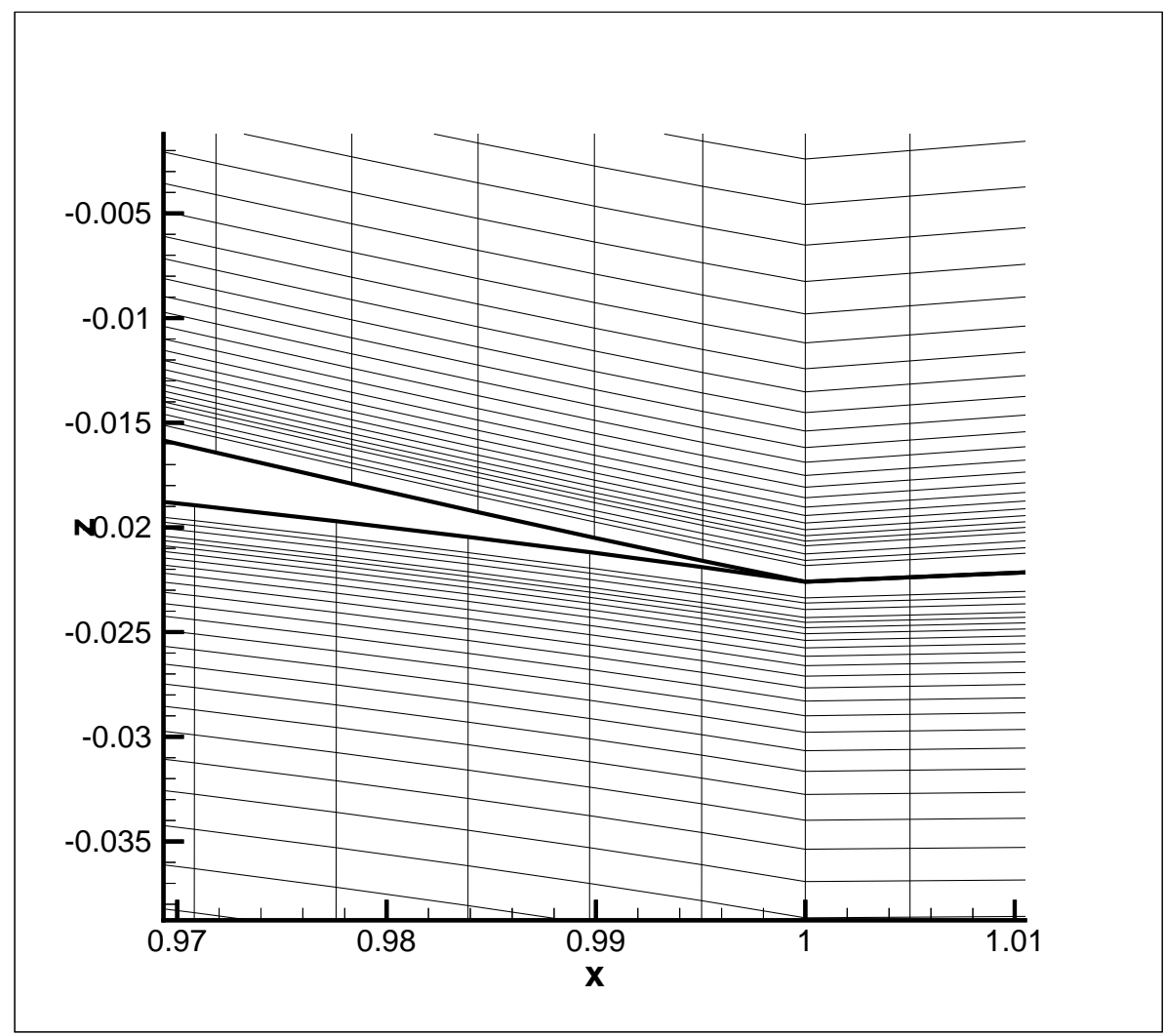

Figure 2: Enlargement of the trailing edge of the mesh

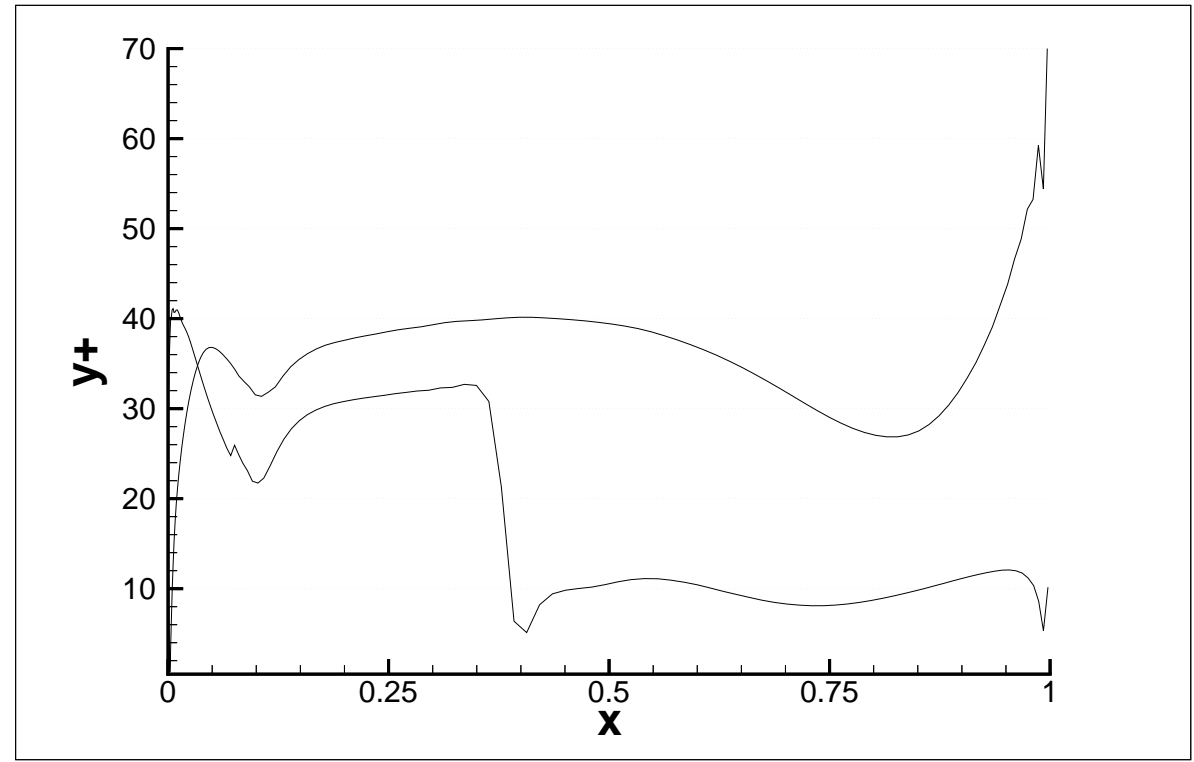

Figure 3: $y^{+}$values of the first cell - $4^{\circ}$ angle of attack - Steady computations 


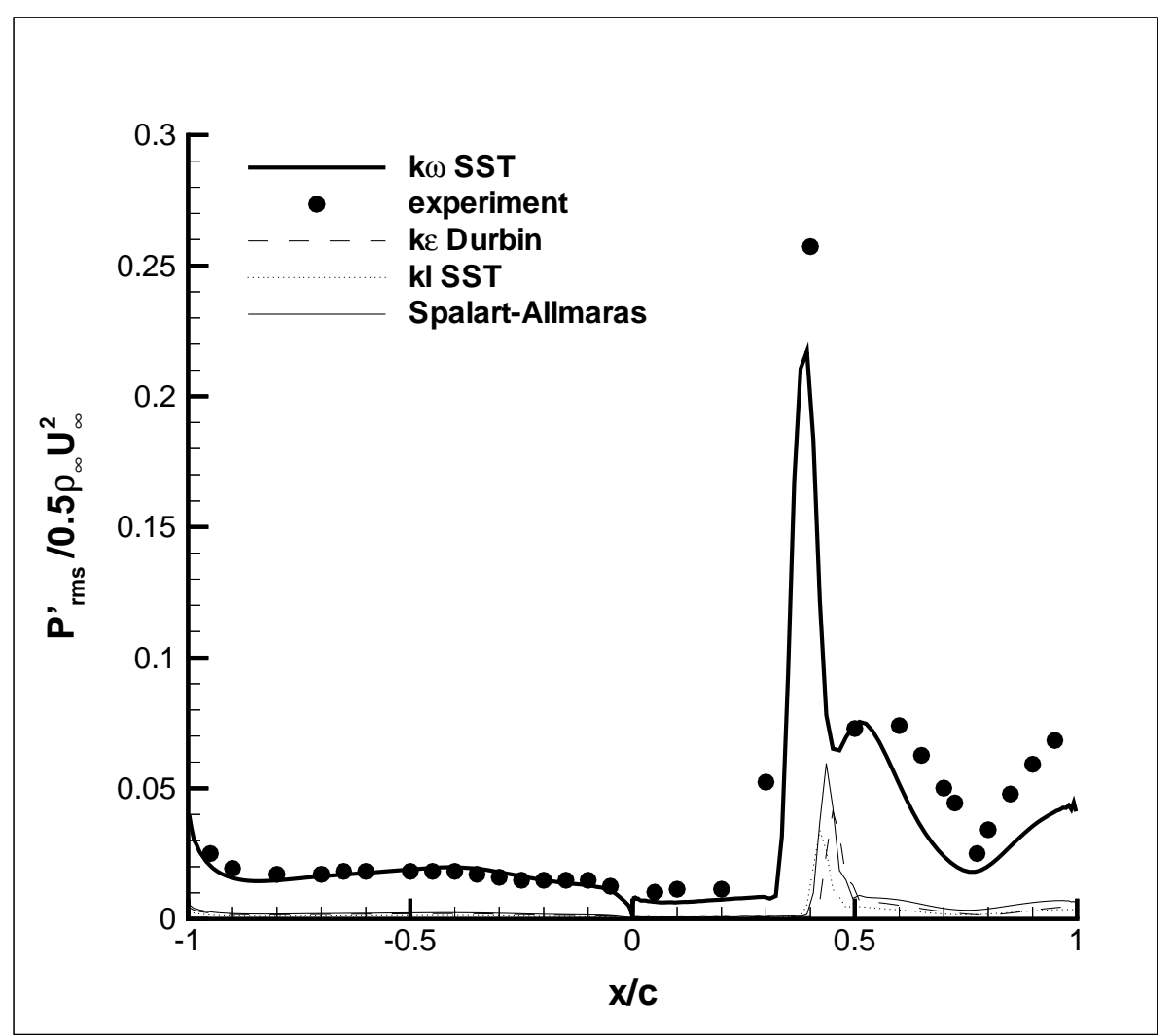

Figure 4: RMS pressure fluctuations over the airfoil - $\alpha=3^{o}$ 


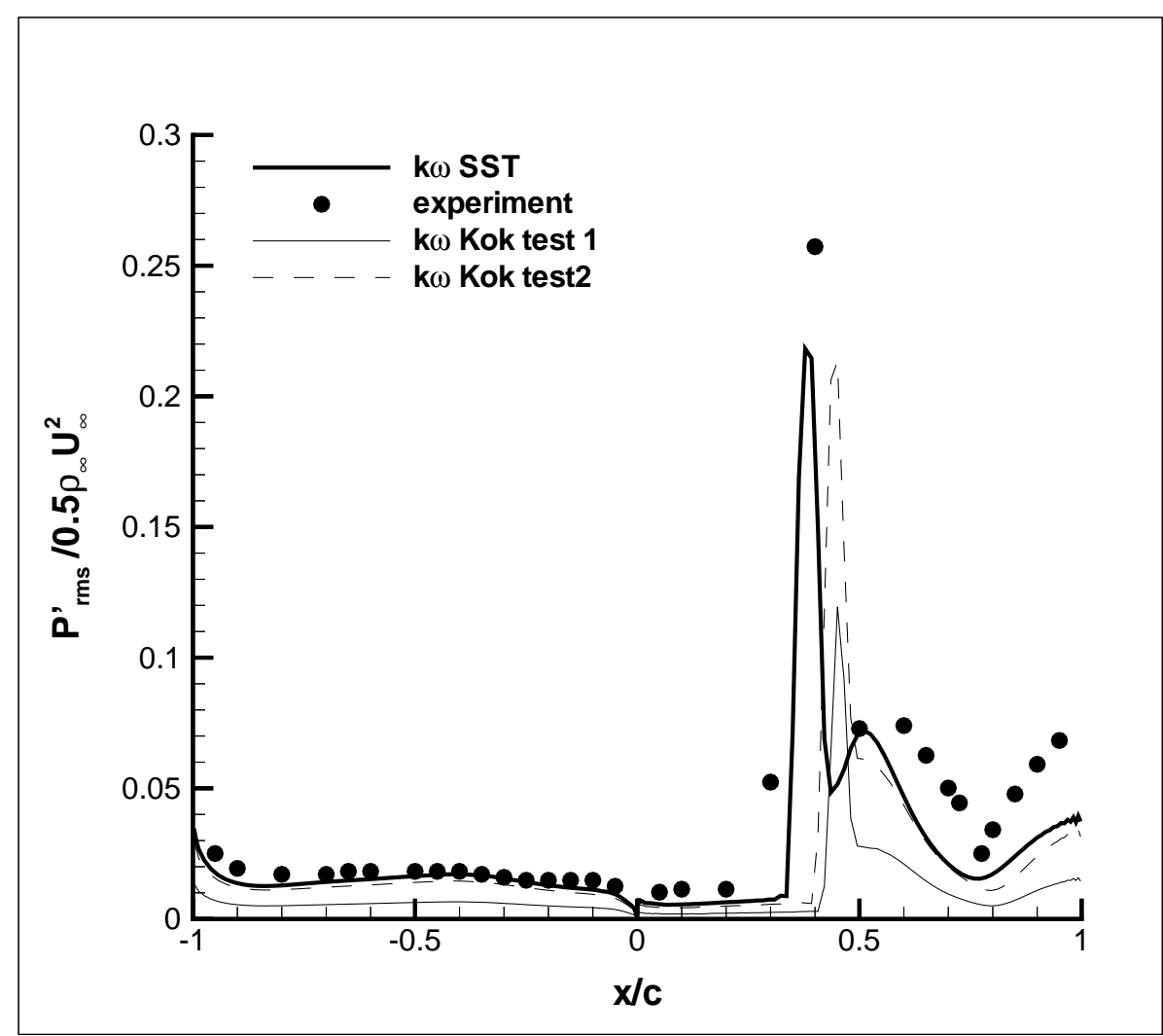

Figure 5: RMS pressure fluctuations over the airfoil $-\alpha=3^{\circ} k-\omega$ models 


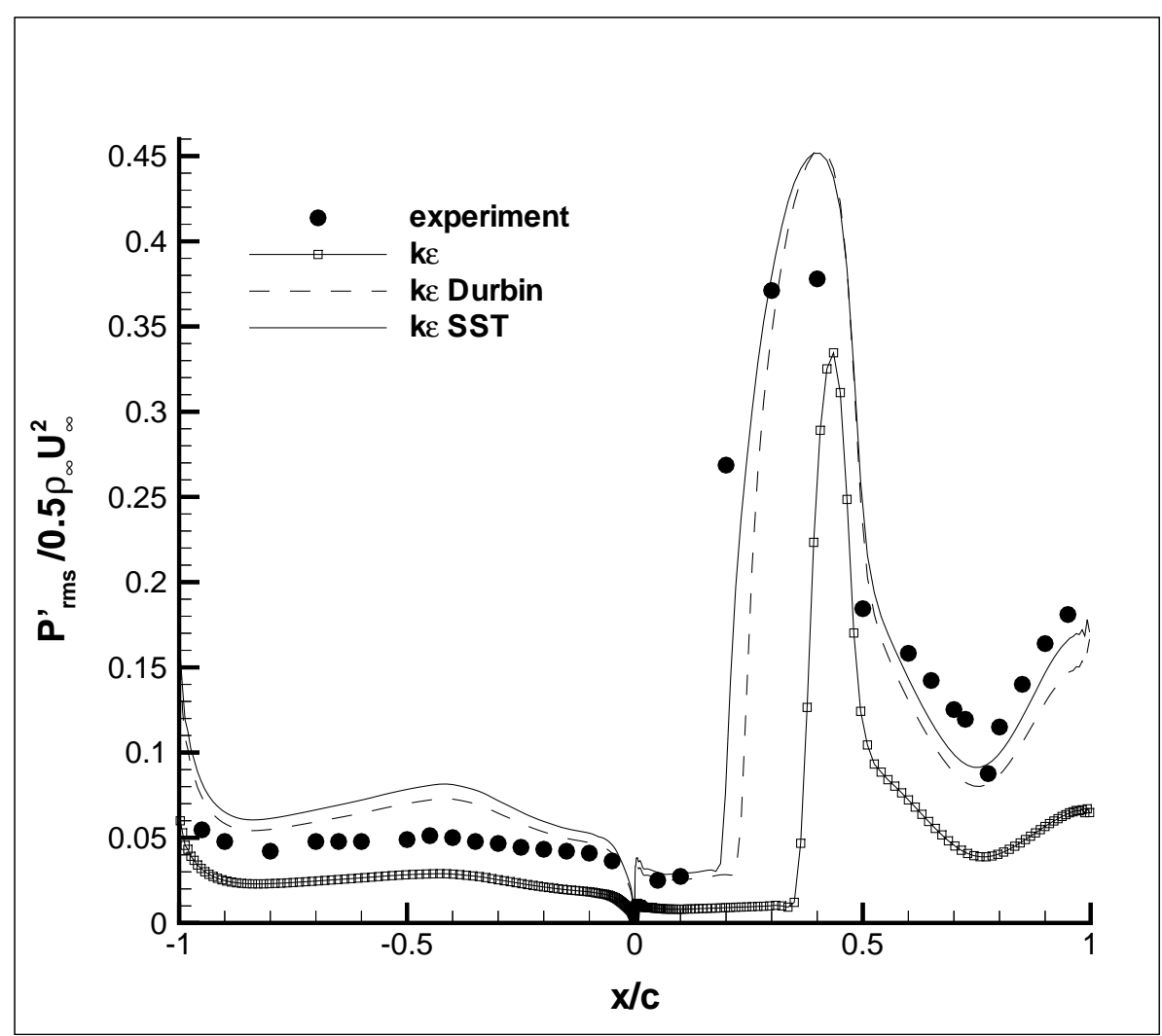

Figure 6: Rms pressure fluctuations over the airfoil - $\alpha=4^{o} k-\varepsilon$ models 


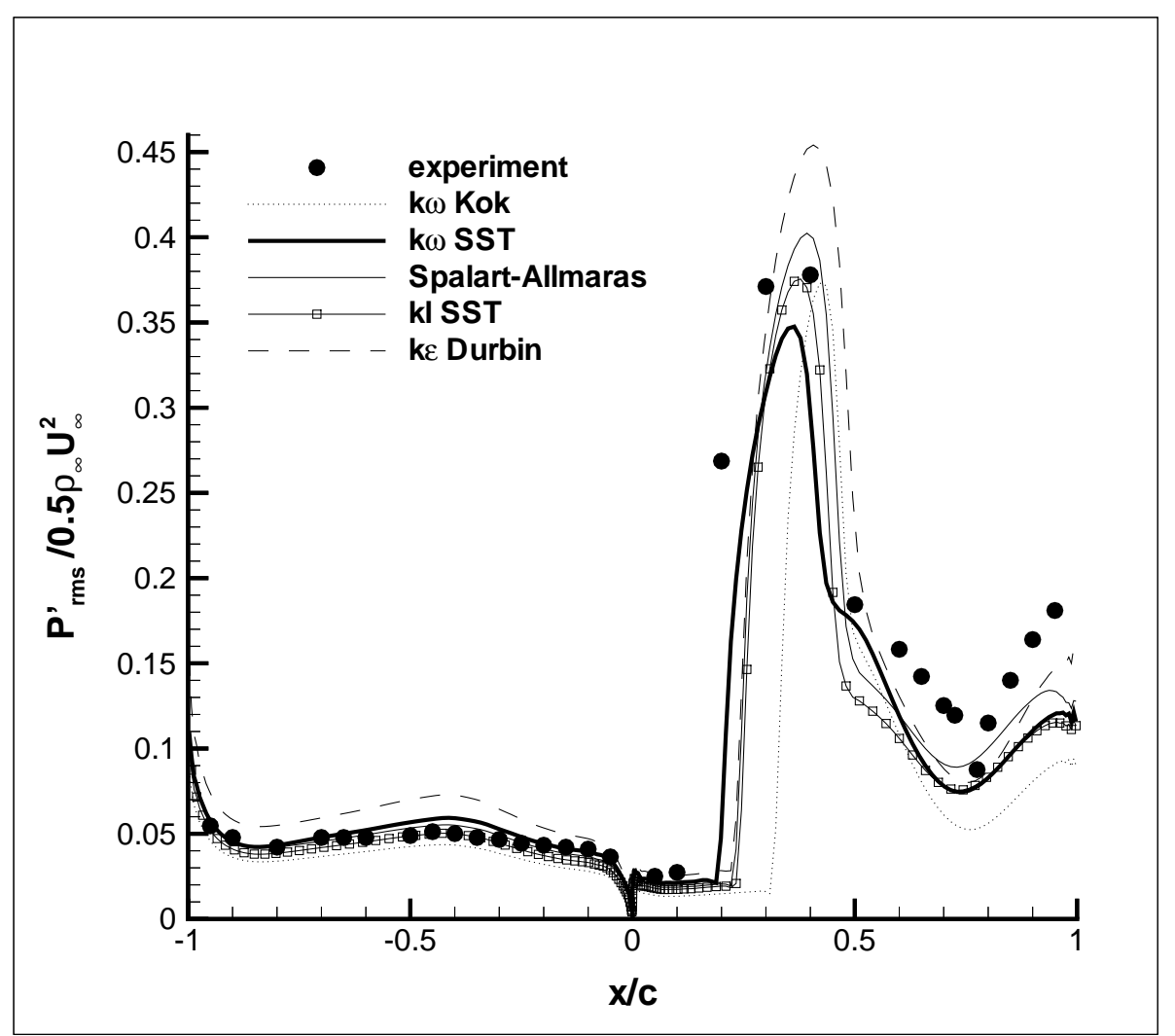

Figure 7: Rms pressure fluctuations over the airfoil - $\alpha=4^{o}$ 


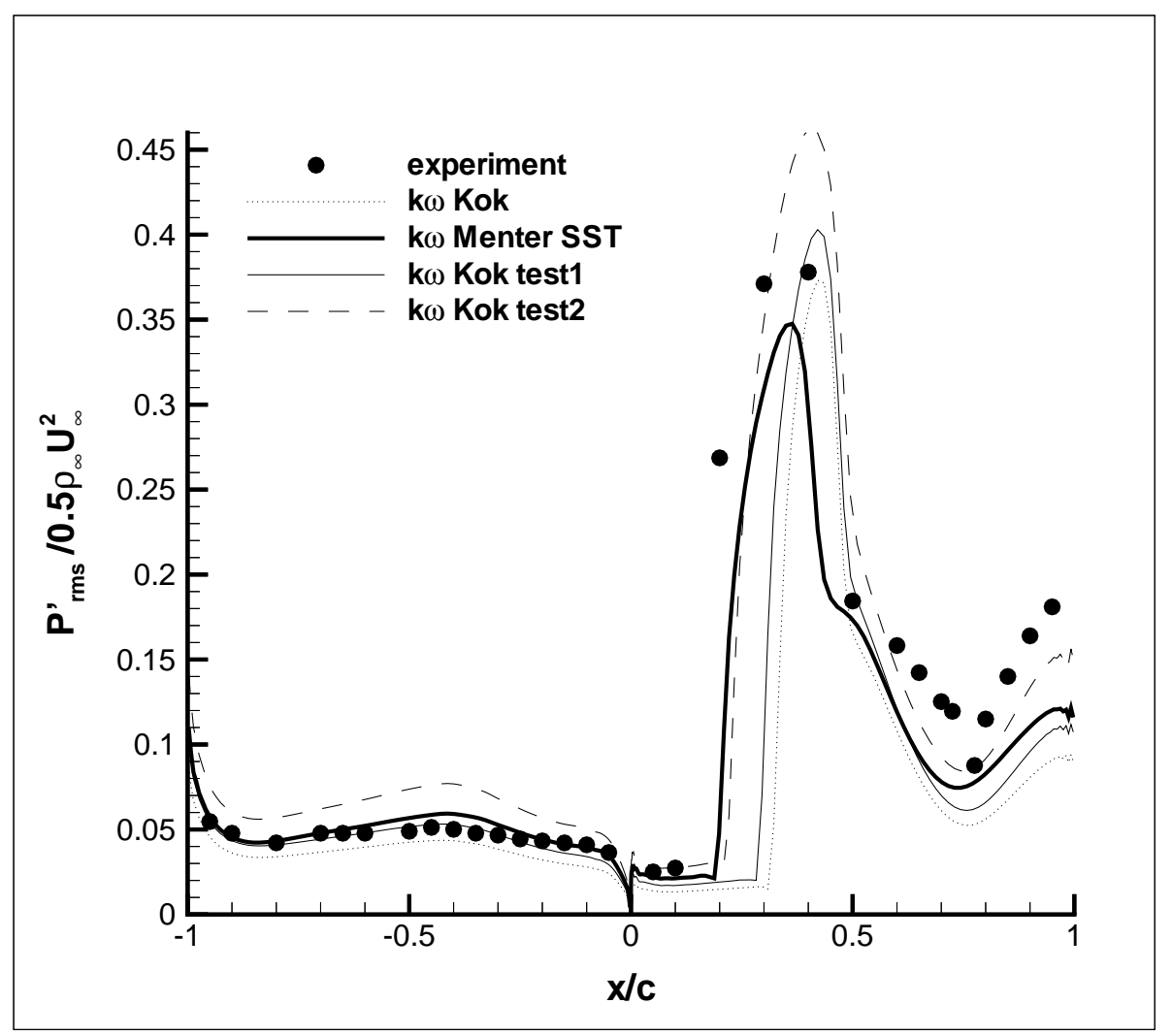

Figure 8: Rms pressure fluctuations over the airfoil - $\alpha=4^{\circ}$ 


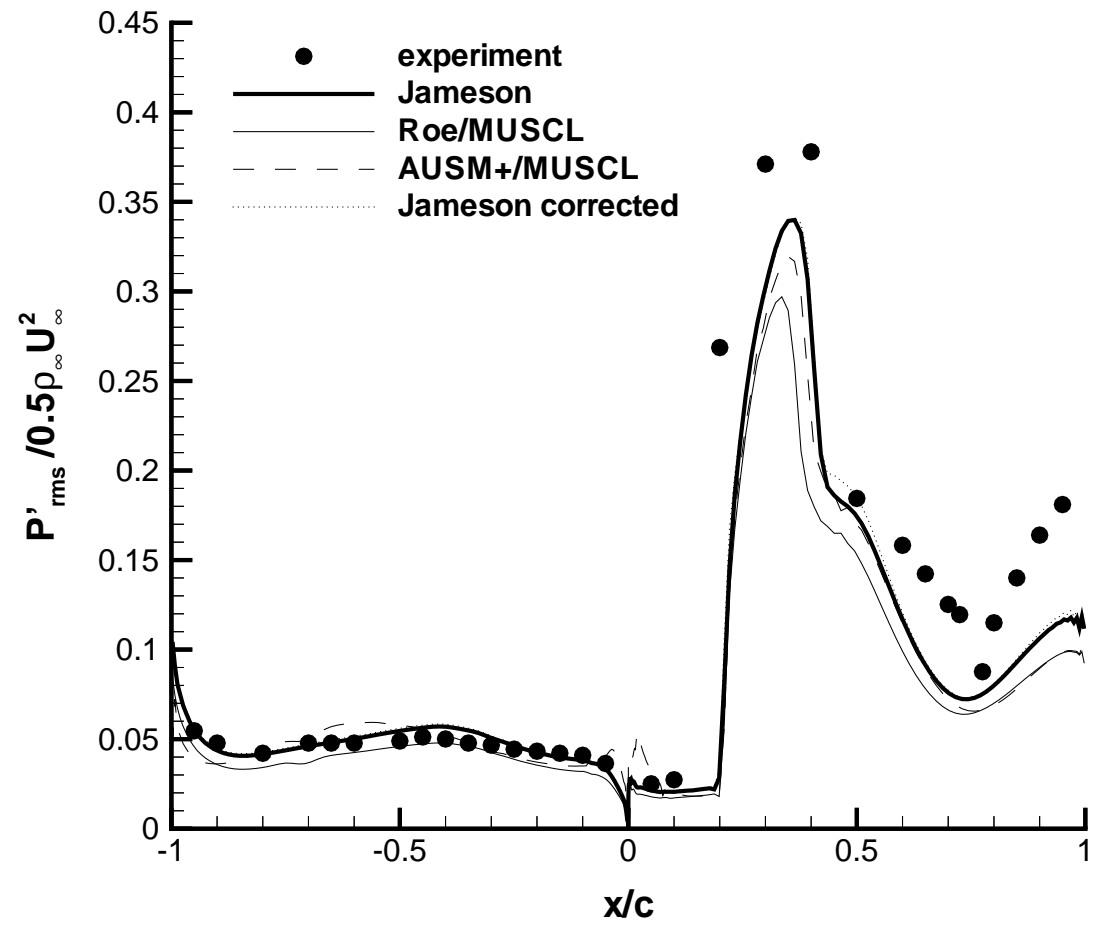

Figure 9: Rms pressure fluctuations over the airfoil $-\alpha=4^{o}$ 


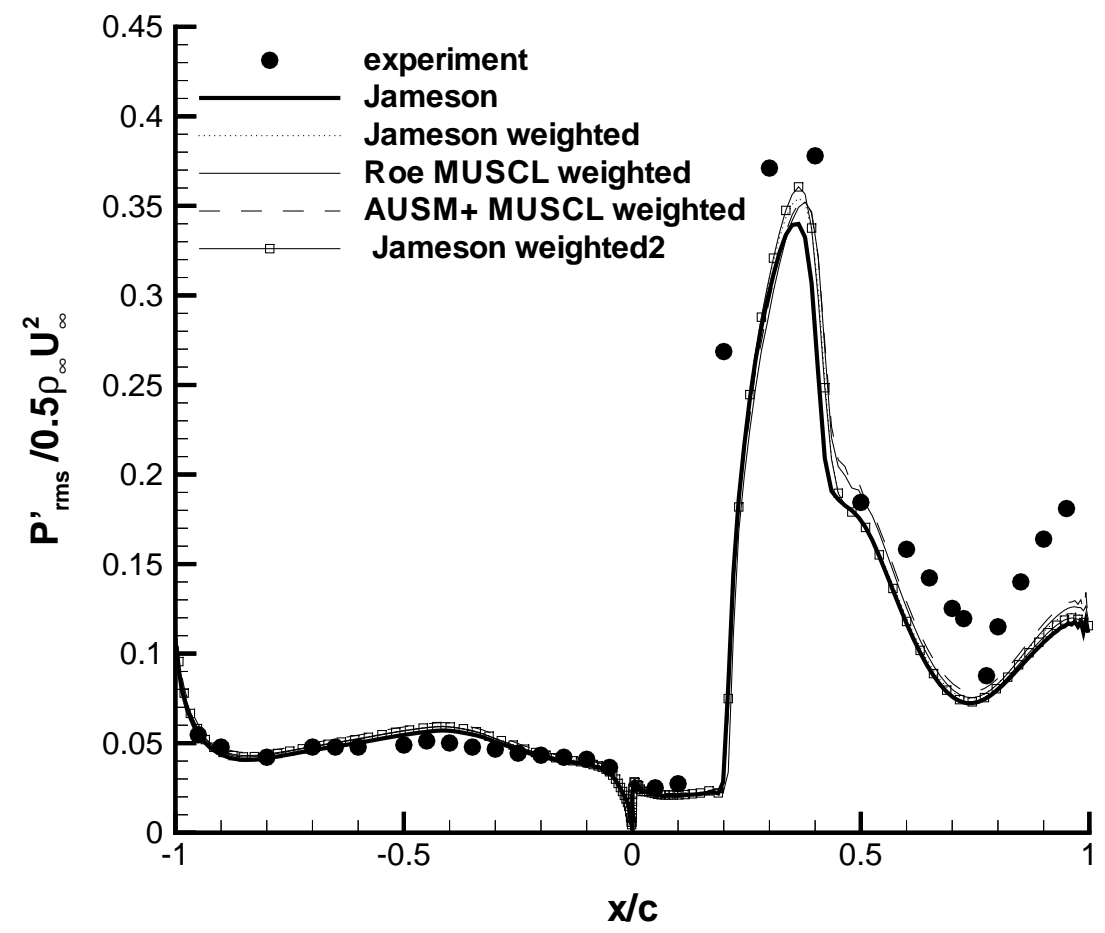

Figure 10: Rms pressure fluctuations over the airfoil $-\alpha=4^{o}$ 


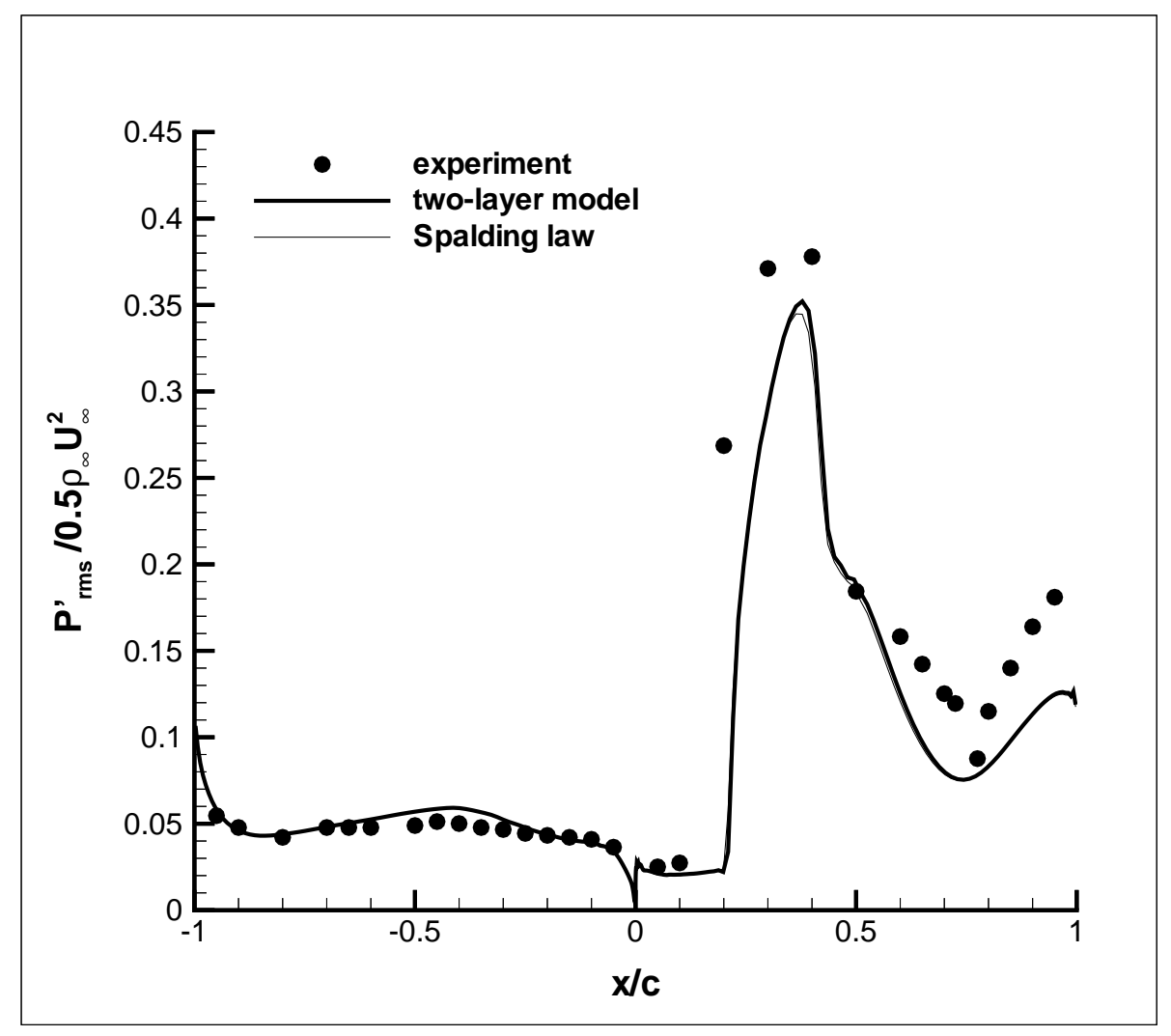

Figure 11: Rms pressure fluctuations over the airfoil - $\alpha=4^{\circ}$ 


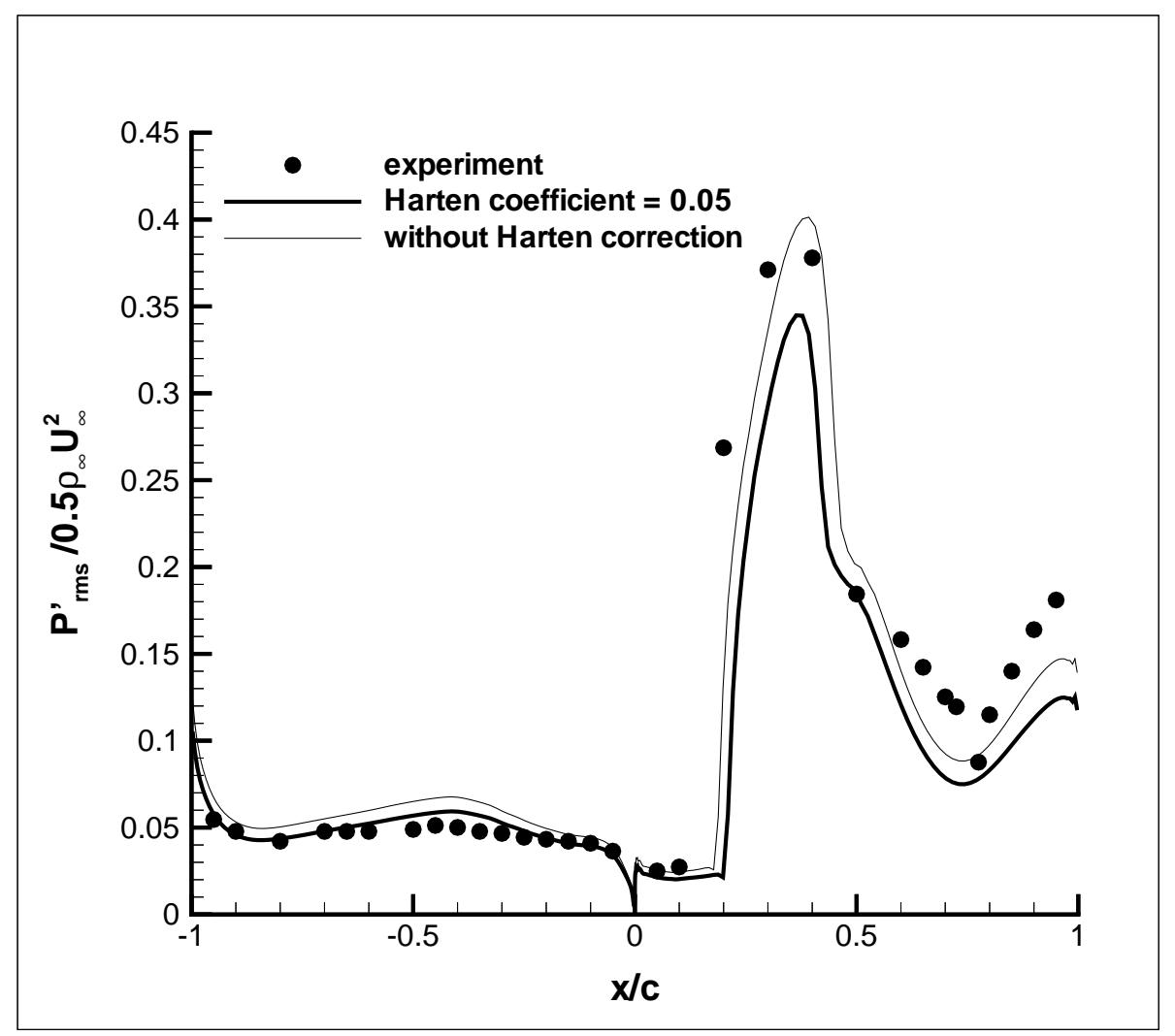

Figure 12: Rms pressure fluctuations over the airfoil - $\alpha=4^{\circ}$ 


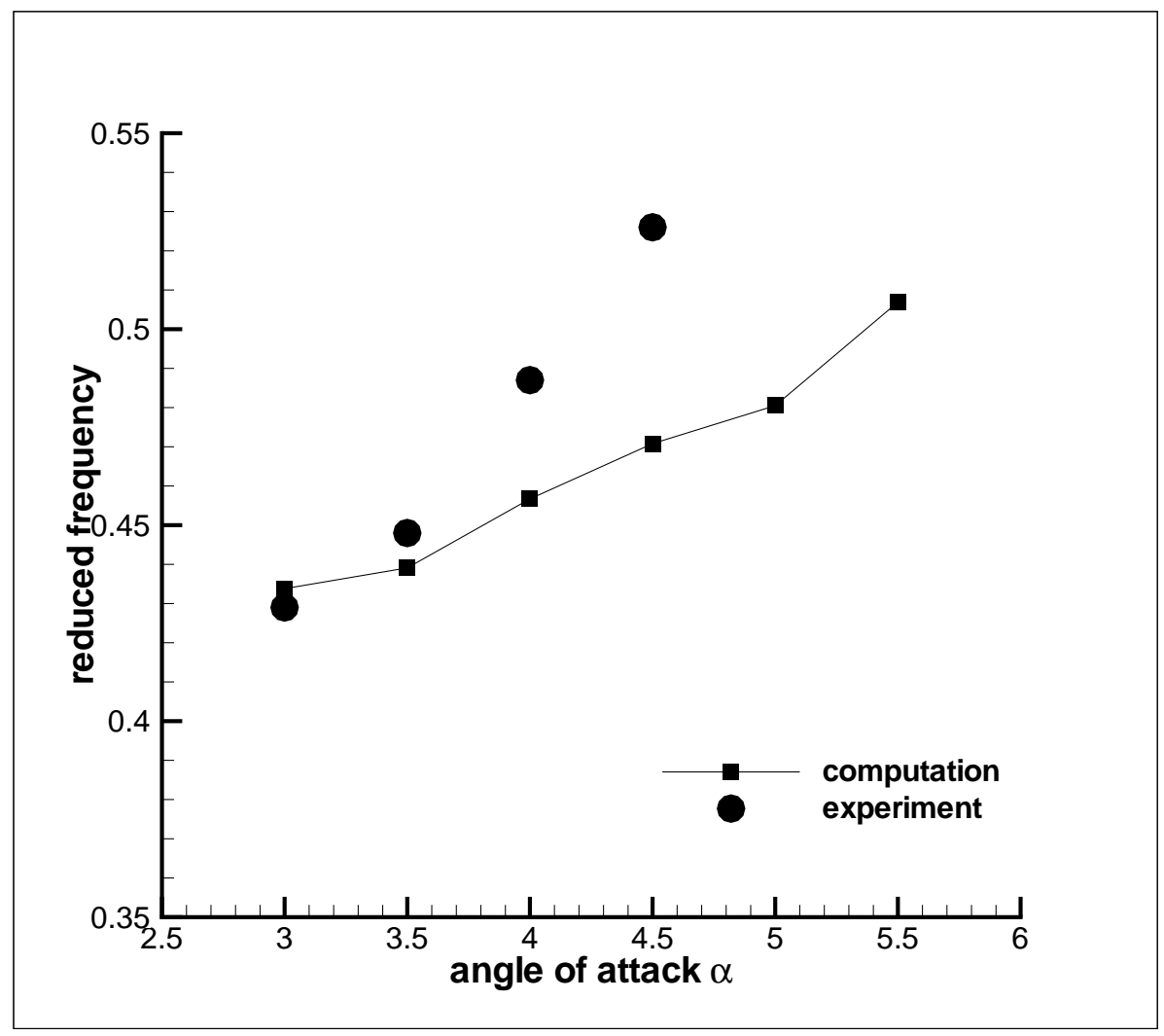

Figure 13: Reduced frequency versus angle of attack 


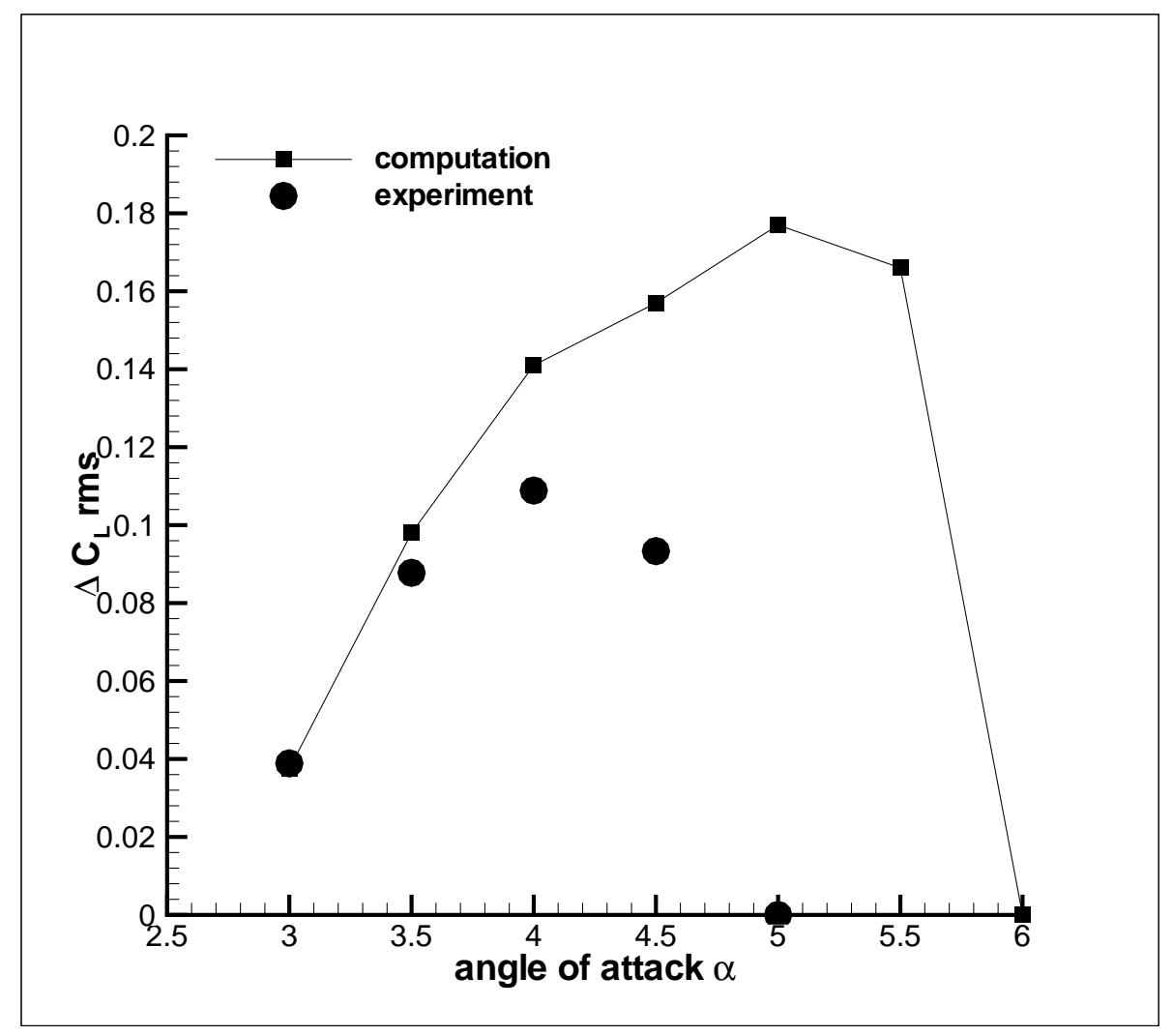

Figure 14: Amplitude rms of the lift coefficient versus angle of attack 


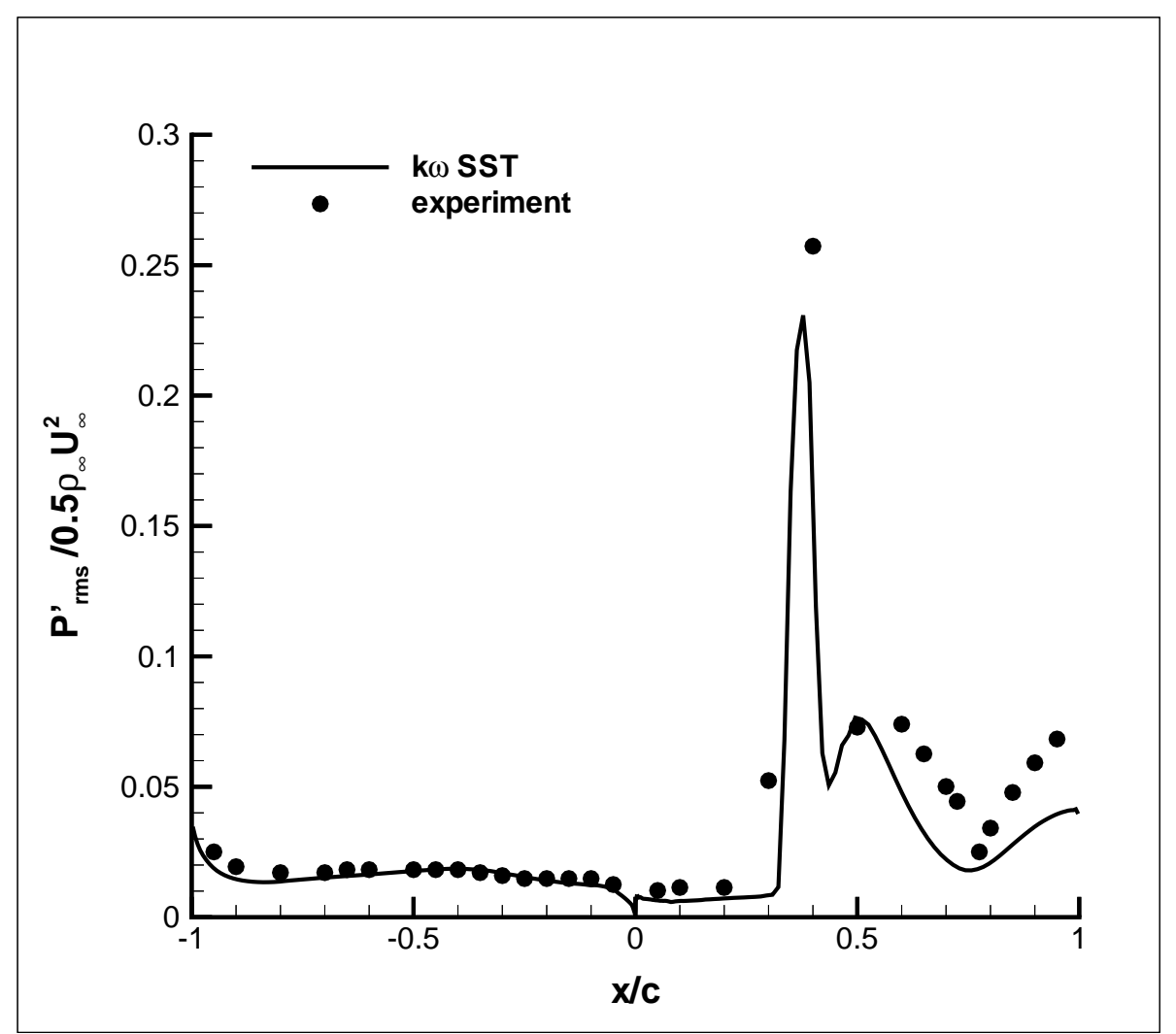

Figure 15: Rms pressure fluctuations over the airfoil $-\alpha=3^{o}$ 


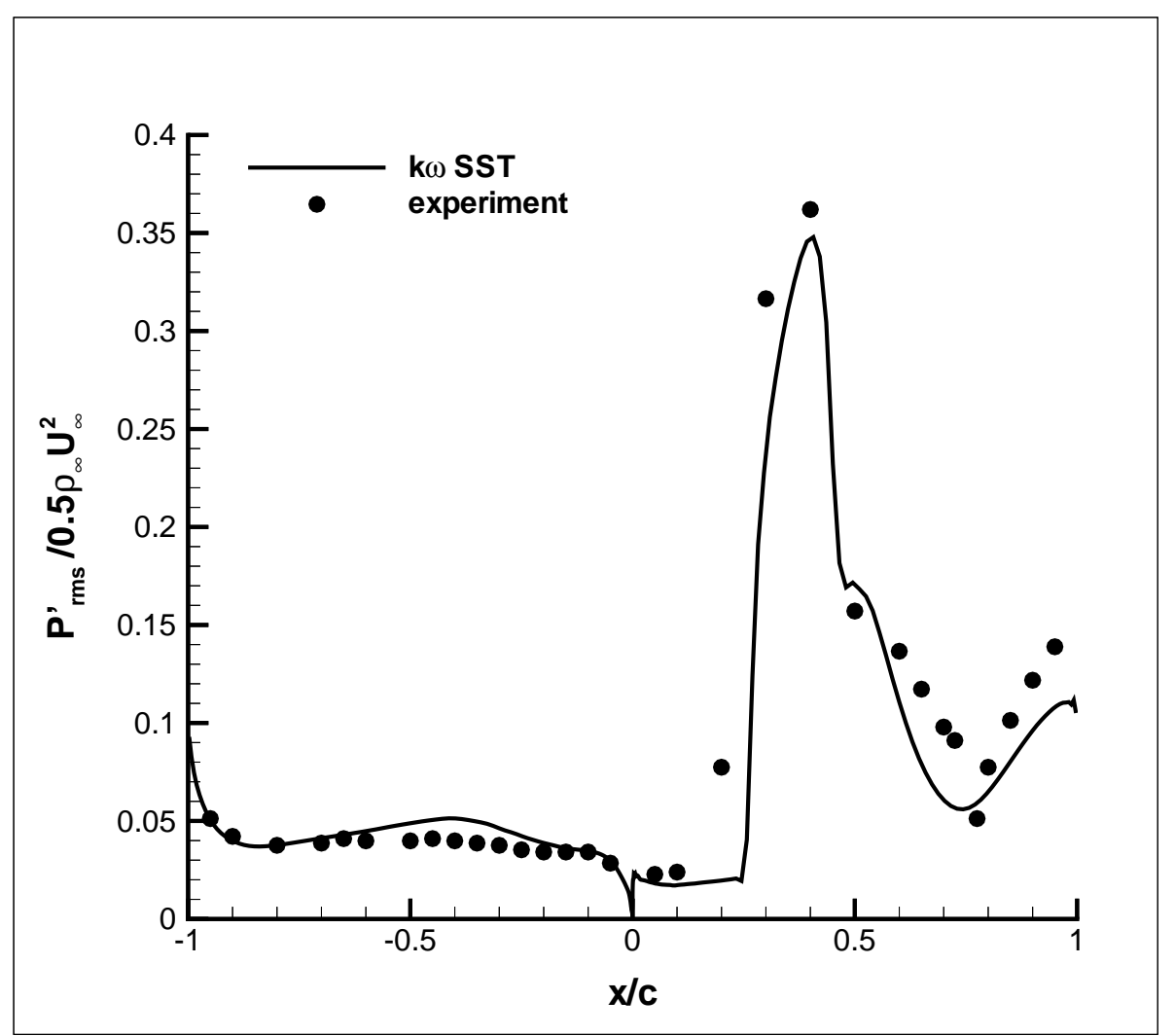

Figure 16: Rms pressure fluctuations over the airfoil $-\alpha=3.5^{\circ}$ 


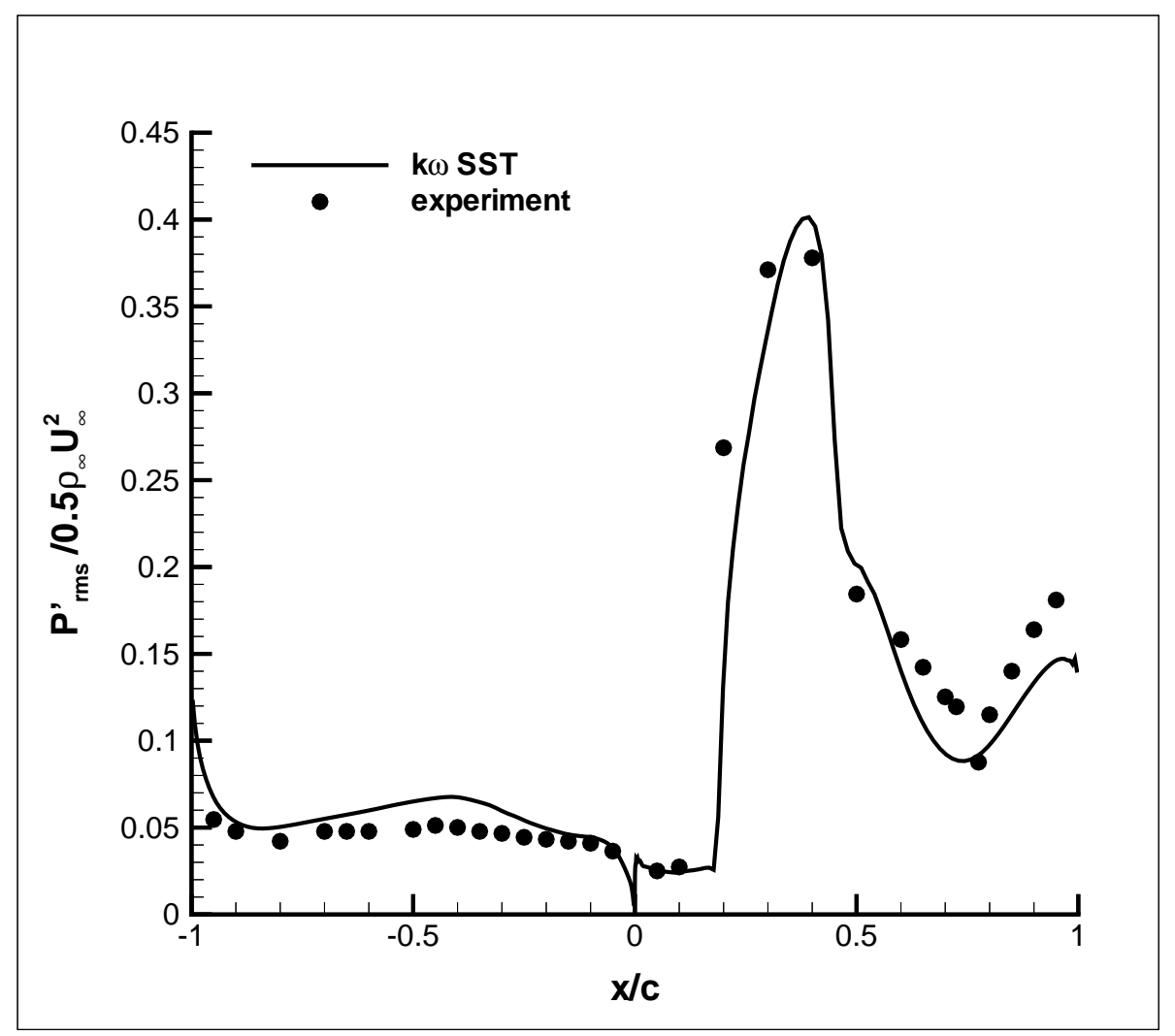

Figure 17: Rms pressure fluctuations over the airfoil - $\alpha=4^{\circ}$ 